\title{
SPHINCS: practical stateless hash-based signatures
}

\author{
Daniel J. Bernstein ${ }^{1,3}$, Daira Hopwood ${ }^{2}$, Andreas Hülsing ${ }^{3}$, Tanja Lange ${ }^{3}$, \\ Ruben Niederhagen ${ }^{3}$, Louiza Papachristodoulou ${ }^{4}$, Michael Schneider, \\ Peter Schwabe ${ }^{4}$, and Zooko Wilcox-O'Hearn ${ }^{2}$ \\ 1 Department of Computer Science, University of Illinois at Chicago, \\ Chicago, IL 60607-7045, USA \\ djb@cr.yp.to \\ 2 Least Authority, 3450 Emerson Ave. Boulder, CO 80305-6452 USA \\ daira@leastauthority.com, zooko@leastauthority.com \\ 3 Department of Mathematics and Computer Science, Technische Universiteit \\ Eindhoven, P.O. Box 513, 5600 MB Eindhoven, The Netherlands \\ tanja@hyperelliptic.org, ruben@polycephaly.org, \\ andreas.huelsing@googlemail.com \\ 4 Radboud University Nijmegen, Digital Security Group, \\ P.O. Box 9010, 6500 GL Nijmegen, The Netherlands \\ louiza@cryptologio.org, peter@cryptojedi.org
}

\begin{abstract}
This paper introduces a high-security post-quantum stateless hash-based signature scheme that signs hundreds of messages per second on a modern 4 -core $3.5 \mathrm{GHz}$ Intel CPU. Signatures are $41 \mathrm{~KB}$, public keys are $1 \mathrm{~KB}$, and private keys are $1 \mathrm{~KB}$. The signature scheme is designed to provide long-term $2^{128}$ security even against attackers equipped with quantum computers. Unlike most hash-based designs, this signature scheme is stateless, allowing it to be a drop-in replacement for current signature schemes.
\end{abstract}

Keywords: post-quantum cryptography, one-time signatures, few-time signatures, hypertrees, vectorized implementation

\section{Introduction}

It is not at all clear how to securely sign operating-system updates, web-site certificates, etc. once an attacker has constructed a large quantum computer:

- RSA and ECC are perceived today as being small and fast, but they are broken in polynomial time by Shor's algorithm. The polynomial is so small that scaling up to secure parameters seems impossible.

This work was supported by the National Science Foundation under grant 1018836 and by the Netherlands Organisation for Scientific Research (NWO) under grant 639.073.005 and Veni 2013 project 13114 and by the European Commission through the ICT program under contract INFSO-ICT-284833 (PUFFIN). Permanent ID of this document: 5c2820cfddf4e259cc7ea1eda384c9f9. Date: 2015.02.02. 
- Lattice-based signature schemes are reasonably fast and provide reasonably small signatures and keys for proposed parameters. However, their quantitative security levels are highly unclear. It is unsurprising for a lattice-based scheme to promise "100-bit" security for a parameter set in 2012 and to correct this promise to only "75-80 bits" in 2013 (see [19, footnote 2]). Furthermore, both of these promises are only against pre-quantum attacks, and it seems likely that the same parameters will be breakable in practice by quantum computers.

- Multivariate-quadratic signature schemes have extremely short signatures, are reasonably fast, and in some cases have public keys short enough for typical applications. However, the long-term security of these schemes is even less clear than the security of lattice-based schemes.

- Code-based signature schemes provide short signatures, and in some cases have been studied enough to support quantitative security conjectures. However, the schemes that have attracted the most security analysis have keys of many megabytes, and would need even larger keys to be secure against quantum computers.

Hash-based signature schemes are perhaps the most attractive answer. Every signature scheme uses a cryptographic hash function; hash-based signatures use nothing else. Many hash-based signature schemes offer security proofs relative to comprehensible, and plausible, properties of the hash function, properties that have not been broken even when the hash function is MD5. (We do not mean to suggest that MD5 is a good choice of hash function; it is easy to make, and we recommend, much more conservative parameter choices.) A recent result by Song [35] shows that these proofs are still valid for quantum adversaries; this is not known to be the case for many other post-quantum signature proposals. Hashbased signing is reasonably fast, even without hardware acceleration; verification is faster; signatures and keys are reasonably small.

However, every practical hash-based signature scheme in the literature is stateful. Signing reads a secret key and a message and generates a signature but also generates an updated secret key. This does not fit standard APIs; it does not even fit the standard definition of signatures in cryptography. If the update fails (for example, if a key is copied from one device to another, or backed up and later restored) then security disintegrates.

It has been known for many years that, as a theoretical matter, one can build hash-based signature schemes without a state. What we show in this paper is that high-security post-quantum stateless hash-based signature systems are practical, and in particular that they can sign hundreds of messages per second on a modern 4 -core $3.5 \mathrm{GHz}$ Intel $\mathrm{CPU}$ using parameters that provide $2^{128}$ security against quantum attacks. In particular, we

- introduce SPHINCS, a new method to do randomized tree-based stateless signatures;

- introduce HORS with trees (HORST), an improvement of the HORS fewtime signature scheme; 
- propose SPHINCS-256, an efficient high-security instantiation of SPHINCS; and

- describe a fast vectorized implementation of SPHINCS-256.

SPHINCS is carefully designed so that its security can be based on weak standardmodel assumptions, avoiding collision resistance and the random-oracle model.

Hash-based Signatures. The idea of hash-based signatures goes back to a proposal from 1979 by Lamport [30]. In Lamport's scheme, the public key consists of two hash outputs for secret inputs; to sign bit 0 , reveal the preimage of the first output; to sign bit 1, reveal the preimage of the second output. Obviously the secret key in this scheme can be used only once: signing a total of $T$ bits of messages requires a sequence of $T$ public keys and is therefore highly impractical.

To allow a short public key to sign many messages, Merkle [32] proposed what are now called Merkle trees. Merkle starts with a one-time signature scheme (OTS), i.e. a signature scheme where a key pair is used only once. To construct a many-time signature scheme, Merkle authenticates $2^{h}$ OTS key pairs using a binary hash tree of height $h$. The leaves of this tree are the hashes of the OTS public keys. The OTS secret keys become the secret key of the new scheme and the root of the tree the public key. A key pair can be used to sign $2^{h}$ messages.

A signature of the many-time signature scheme is also called a full signature if necessary to distinguish it from other kinds of signatures. A full signature contains the index of the used OTS key pair in the tree; the OTS public key; the OTS signature; and the authentication path, i.e., the set of sibling nodes on the path from the OTS public key to the root. (If a Winternitz-style OTS is used, the OTS public key can be computed from the OTS signature. Hence, the OTS public key can be omitted in the full signature in that case.) To guarantee that each OTS key pair is used only once, the OTS key pairs are used in a predefined order, using the leaves of the tree from left to right. To verify the signature, one verifies the OTS signature on the message, and verifies the authenticity of the OTS key pair by checking whether the public key is consistent with the authentication path and the hash of the OTS public key.

This approach generates small signatures, small secret keys (using pseudorandom generation of the OTS secret keys), and small public keys. However, key generation and signature time are exponential in $h$ as the whole tree has to be built in the key generation. Recent practical hash-based signature systems $[27,15,18,16,17]$ solve these two performance problems. First, key generation time is significantly reduced using a hyper-tree of several layers of trees, i.e. a certification tree where a single hash tree of height $h_{1}$ is used to sign the public keys of $2^{h_{1}}$ hash-based key pairs and so on. During key generation only one tree on each layer has to be generated. Using $d$ layers of trees with height $h / d$ reduces the key-generation time from $\mathcal{O}\left(2^{h}\right)$ to $\mathcal{O}\left(d 2^{h / d}\right)$. Second, signing time is reduced from $\mathcal{O}\left(2^{h}\right)$ to $\mathcal{O}(h)$ using stateful algorithms that exploit the ordered use of the OTS key pairs. When combined with hyper-trees, the ordered use of the trees reduces the signing time even further to $\mathcal{O}(h / d)$.

From Stateful to Stateless. Goldreich [23] (elaborating upon [22]) proposed a stateless hash-based signature scheme, using a binary certification tree built 
out of one-time signature keys. In Goldreich's system, each OTS key pair corresponding to a non-leaf node is used to sign the hash of the public keys of its two child nodes. The leaf OTS key pairs are used to sign the messages. The OTS public key of the root becomes the overall public key. The secret key is a seed value that is used to pseudorandomly generate all the OTS key pairs of the tree.

It is important to ensure that a single OTS key pair is never used to sign two different messages. One approach is to sign message $M$ using a tree of height $n$ as follows: compute an $n$-bit hash of $M$, view the hash as an integer $h$ between 0 and $2^{n}-1$, and use the leaf at index $h$ to sign. The full signature contains all the OTS public keys in the path from leaf $h$ to the root, all the public keys of the sibling nodes on this path, and the one-time signatures on the message and on the public keys in the path. Security obviously cannot be better than the collision resistance of the hash function, at most $2^{n / 2}$.

For this scheme, key generation requires a single OTS key generation. Signing takes $2 n$ OTS key generations and $n$ OTS signatures. This can be done in reasonable time for secure parameters. Keys are also very short: one OTS public key $\left(\mathcal{O}\left(n^{2}\right)\right)$ for the public key and a single seed value $(\mathcal{O}(n))$ for the secret key. However, the signature size is cubic in the security parameter. Consider, for example, the Winternitz OTS construction from [26], which has small signatures for a hash-based OTS; taking $n=256$ as we do for SPHINCS-256, and applying some straightforward optimizations, produces a Goldreich signature size that is still above $1 \mathrm{MB}$.

One way to evaluate the real-world impact of particular signature sizes is to compare those sizes to the sizes of messages being signed. For example, in the Debian operating system (September 2014 Wheezy distribution), the average package size is $1.2 \mathrm{MB}$ and the median package size is just $0.08 \mathrm{MB}$. Debian is designed for frequent updates, typically upgrading just one package or a few packages at a time, and of course each upgrade has to check at least one new signature. As another example, the size of an average web page in the Alexa Top 1000000 is $1.8 \mathrm{MB}$, and HTTPS typically sends multiple signatures per page; the exact number depends on how many HTTPS sites cooperate to produce the page, how many certificates are sent, etc. A signature size above $1 \mathrm{MB}$ would often dominate the traffic in these applications and would also add user-visible latency on typical network connections.

Goldreich also proposes randomized leaf selection: instead of applying a public hash function to the message to determine the index of the OTS key pair, select an index randomly. It is then safe for the total tree height $h$ to be somewhat smaller than the hash output length $n$ : the hash output length protects against offline computations by the attacker, while the tree height protects against accidental collisions in indices chosen by the signer. For example, choosing $h$ as 128 instead of 256 saves a factor of 2 in signature size and signing speed, if it is acceptable to have probability roughly $2^{-30}$ of OTS reuse (presumably breaking the system) within $2^{50}$ signatures.

The SPHINCS Approach. SPHINCS introduces two new ideas that together drastically reduce signature size. First, to increase the security level of random- 
ized index selection, SPHINCS replaces the leaf OTS with a hash-based few-time signature scheme (FTS). An FTS is, as the name suggests, a signature scheme designed to sign a few messages; in the context of SPHINCS this allows a few index collisions, which in turn allows a smaller tree height for the same security level. For our FTS (see below) the probability of a forgery after $\gamma$ signatures gradually increases with $\gamma$, while the probability that the signer uses the same FTS key $\gamma$ times gradually decreases with $\gamma$; we choose parameters to make sure that the product of these probabilities is sufficiently small for all $\gamma \in \mathbb{N}$. For example, SPHINCS-256 reduces the total tree height from 256 to just 60 while maintaining $2^{128}$ security against quantum attackers.

Second, SPHINCS views Goldreich's construction as a hyper-tree construction with $h$ layers of trees of height 1 , and generalizes to a hyper-tree with $d$ layers of trees of height $h / d$. This introduces a tradeoff between signature size and time controlled by the number of layers $d$. The signature size is $|\sigma| \approx d\left|\sigma_{\text {OTS }}\right|+h n$ assuming a hash function with $n$-bit outputs. Recall that the size of a one-time signature $\left|\sigma_{\text {OTS }}\right|$ is roughly $\mathcal{O}\left(n^{2}\right)$, so by decreasing the number of layers we get smaller full signatures. The tradeoff is that signing time increases exponentially in the decrease of layers: signing takes $d 2^{h / d}$ OTS key generations and $d 2^{h / d}-d$ hash computations. For example, in SPHINCS-256, with $h=60$, we reduce $d$ from 60 to 12 , increasing $d 2^{h / d}$ from 120 to 384 .

We accompany our construction with an exact security reduction to some standard-model properties of hash functions. For parameter selection, we analyze the costs of generic attacks against these properties when the attacker has access to a large-scale quantum computer. For SPHINCS-256 we select parameters that provide 128 bits of security against quantum attackers and keep a balance between signature size and time.

HORS and HORST. HORS [34] is a fast hash-based FTS. For message hashes of length $m$, HORS uses two parameters $t=2^{\tau}$ for $\tau \in \mathbb{N}$ and $k \in \mathbb{N}$ such that $m=k \log t=k \tau$. For practical secure parameters $t \gg k$. HORS uses a secret key consisting of $t$ random values. The public key consists of the $t$ hashes of these values. A signature consists of $k$ secret key elements, with indices selected as public functions of the message being signed.

In the context of SPHINCS, each full signature has to include not just an FTS signature but also an FTS public key. The problem with HORS is that it has large public keys. Of course, one can replace the public key in any signature system by a short hash of the original public key, but then the original public key needs to be included in the signature; this does not improve the total length of key and signature.

As a better FTS for SPHINCS we introduce HORS with trees (HORST). Compared to HORS, HORST sacrifices runtime to reduce the public key size and the combined size of a signature and a public key. A HORST public key is the root node of a binary hash tree of height $\log t$, where the leaves are the public key elements of a HORS key. This reduces the public key size to a single hash value. For this to work, a HORST signature contains not only the $k$ secret key elements but also one authentication path per secret key element. Now the public 
key can be computed given a signature. A full hash-based signature thus includes just $k \log t$ hash values for HORST, compared to $t$ hash values for HORS. We also introduce some optimizations that further compress signatures.

For the SPHINCS-256 parameters, switching from HORS to HORST reduces the FTS part of the full signature from $2^{16}$ hash values to fewer than $16 \cdot 32=2^{9}$ hash values, i.e., from $2 \mathrm{MB}$ to just $16 \mathrm{~KB}$.

The same idea applies in more generality. For example, HORS $++[33]$ is a variant of HORS that gives stronger security guarantees but that has bigger keys; the changes from HORS to HORST can easily be adapted to $\mathrm{HORS}++$, producing HORST ++ .

Vectorized Software Implementation. We also present an optimized implementation of SPHINCS-256. Almost all hash computations in SPHINCS are highly parallel and we make extensive use of vector instructions. On an Intel Xeon E3-1275 (Haswell) CPU, our hashing throughput is about 1.6 cycles/byte. Signing a short message with SPHINCS-256 takes 51636372 cycles on a single core; simultaneous signing on all 4 cores of the $3.5 \mathrm{GHz} \mathrm{CPU}$ has a throughput of more than 200 signatures per second, fast enough for most applications. Verification takes only 1451004 cycles; key-pair generation takes 3237260 cycles.

We placed the software described in this paper into the public domain to maximize reusability of our results. We submitted the software to eBACS [10] for independent benchmarking; the software is also available online at http: //cryptojedi.org/crypto/\#sphincs.

Notation. We always use the logarithm with base 2 and hence write log instead of $\log _{2}$. We write $[x]$ for the set $\{0,1, \ldots, x\}$. Given a bit string $x$ we write $x(i)$ for the $i$ th bit of $x$ and $x(i, j)$ for the $j$-bit substring of $x$ that starts with the ith bit.

\section{The SPHINCS Construction}

In this section we describe our main construction. We begin by listing the parameters used in the construction, reviewing the one-time signature scheme WOTS $^{+}$, and reviewing binary hash trees. In Section 2.1 we present our few-time signature scheme HORST, and in Section 2.2 we present our many-time signature scheme SPHINCS.

Parameters. SPHINCS uses several parameters and several functions. The main security parameter is $n \in \mathbb{N}$. The functions include two short-input cryptographic hash functions $\mathrm{F}:\{0,1\}^{n} \rightarrow\{0,1\}^{n}$ and $\mathrm{H}:\{0,1\}^{2 n} \rightarrow\{0,1\}^{n}$; one arbitrary-input randomized hash function $\mathcal{H}:\{0,1\}^{n} \times\{0,1\}^{*} \rightarrow\{0,1\}^{m}$, for $m=\operatorname{poly}(n)$; a family of pseudorandom generators $\mathrm{G}_{\lambda}:\{0,1\}^{n} \rightarrow\{0,1\}^{\lambda n}$ for different values of $\lambda$; an ensemble of pseudorandom function families $\mathcal{F}_{\lambda}$ : $\{0,1\}^{\lambda} \times\{0,1\}^{n} \rightarrow\{0,1\}^{n}$; and a pseudorandom function family $\mathcal{F}:\{0,1\}^{*} \times$ $\{0,1\}^{n} \rightarrow\{0,1\}^{2 n}$ that supports arbitrary input lengths. Of course, these functions can all be built from a single cryptographic hash function, but it is more natural to separate the functions according to their roles. 
SPHINCS uses a hyper-tree (a tree of trees) of total height $h \in \mathbb{N}$, where $h$ is a multiple of $d$ and the hyper-tree consists of $d$ layers of trees, each having height $h / d$. The components of SPHINCS have additional parameters which influence performance and size of the signature and keys: the Winternitz one-time signature WOTS naturally allows for a space-time tradeoff using the Winternitz parameter $w \in \mathbb{N}, w>1$; the tree-based few-time signature scheme HORST has a space-time tradeoff which is controlled by two parameters $k \in \mathbb{N}$ and $t=2^{\tau}$ with $\tau \in \mathbb{N}$ and $k \tau=m$.

As a running example we present concrete numbers for SPHINCS-256; the choices are explained in Section 4. For SPHINCS-256 we use $n=256, m=$ $512, h=60, d=12, w=16, t=2^{16}, k=32$.

WOTS $^{+}$. We now describe the Winternitz one-time signature $\left(\mathrm{WOTS}^{+}\right.$) from [26]. We deviate slightly from the description in [26] to describe the algorithms as they are used in SPHINCS. Specifically, we include pseudorandom key generation and fix the message length to be $n$, meaning that a seed value takes the place of a secret key in our description. Given $n$ and $w$, we define

$$
\ell_{1}=\left\lceil\frac{n}{\log (w)}\right\rceil, \quad \ell_{2}=\left\lfloor\frac{\log \left(\ell_{1}(w-1)\right)}{\log (w)}\right\rfloor+1, \quad \ell=\ell_{1}+\ell_{2} .
$$

For the SPHINCS-256 parameters this leads to $\ell=67$. WOTS ${ }^{+}$uses the function $\mathrm{F}$ to construct the following chaining function.

Chaining function $c^{i}(x, \mathbf{r})$ : On input of value $x \in\{0,1\}^{n}$, iteration counter $i \in \mathbb{N}$, and bitmasks $\mathbf{r}=\left(r_{1}, \ldots, r_{j}\right) \in\{0,1\}^{n \times j}$ with $j \geq i$, the chaining function works the following way. In case $i=0, c$ returns $x$, i.e., $c^{0}(x, \mathbf{r})=x$. For $i>0$ we define $c$ recursively as

$$
c^{i}(x, \mathbf{r})=\mathrm{F}\left(c^{i-1}(x, \mathbf{r}) \oplus r_{i}\right),
$$

i.e. in every round, the function first takes the bitwise xor of the previous value $c^{i-1}(x, \mathbf{r})$ and bitmask $r_{i}$ and evaluates $\mathrm{F}$ on the result. We write $\mathbf{r}_{a, b}$ for the substring $\left(r_{a}, \ldots, r_{b}\right)$ of $\mathbf{r}$. In case $b<a$ we define $\mathbf{r}_{a, b}$ to be the empty string. Now we describe the three algorithms of $\mathrm{WOTS}^{+}$.

Key Generation Algorithm (sk, pk $\leftarrow$ WOTS.kg $(\mathcal{S}, \mathbf{r}))$ : On input of seed $\mathcal{S} \in$ $\{0,1\}^{n}$ and bitmasks $\mathbf{r} \in\{0,1\}^{n \times(w-1)}$ the key generation algorithm computes the internal secret key as $\mathrm{sk}=\left(\mathrm{sk}_{1}, \ldots, \mathrm{sk}_{\ell}\right) \leftarrow \mathrm{G}_{\ell}(\mathcal{S})$, i.e., the $n$ bit seed is expanded to $\ell$ values of $n$ bits. The public key pk is computed as

$$
\mathrm{pk}=\left(\mathrm{pk}_{1}, \ldots, \mathrm{pk}_{\ell}\right)=\left(c^{w-1}\left(\mathrm{sk}_{1}, \mathbf{r}\right), \ldots, c^{w-1}\left(\mathrm{sk}_{\ell}, \mathbf{r}\right)\right)
$$

Note that $\mathcal{S}$ requires less storage than sk; thus we generate sk and pk on the fly when necessary.

Signature Algorithm $(\sigma \leftarrow$ WOTS.sign $(M, \mathcal{S}, \mathbf{r}))$ : On input of an $n$-bit message $M$, seed $\mathcal{S}$ and the bitmasks $\mathbf{r}$, the signature algorithm first computes a base$w$ representation of $M: M=\left(M_{1} \ldots M_{\ell_{1}}\right), M_{i} \in\{0, \ldots, w-1\}$. That is, $M$ 


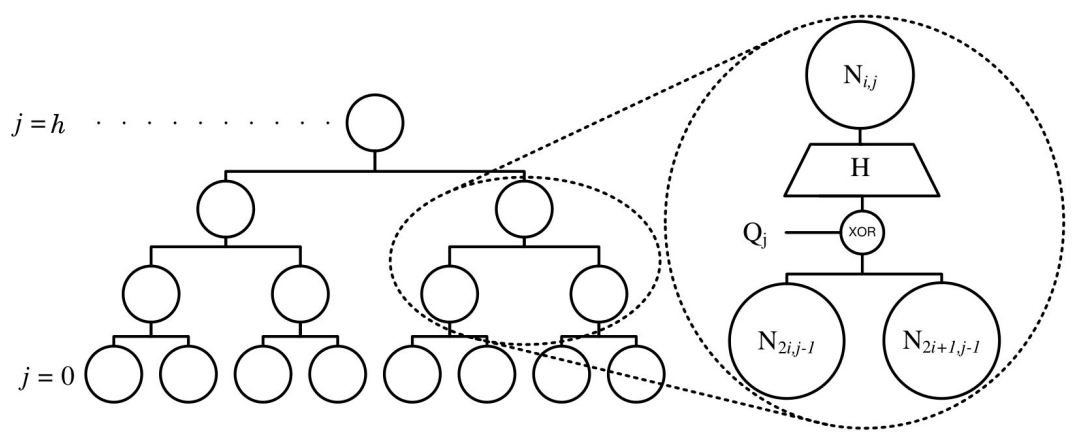

Fig. 1. The binary hash tree construction

is treated as the binary representation of a natural number $x$ and then the $w$-ary representation of $x$ is computed. Next it computes the checksum $C=$ $\sum_{i=1}^{\ell_{1}}\left(w-1-M_{i}\right)$ and its base $w$ representation $C=\left(C_{1}, \ldots, C_{\ell_{2}}\right)$. The length of the base $w$ representation of $C$ is at most $\ell_{2}$ since $C \leq \ell_{1}(w-1)$. We set $B=\left(b_{1}, \ldots, b_{\ell}\right)=M \| C$, the concatenation of the base $w$ representations of $M$ and $C$. Then the internal secret key is generated using $\mathrm{G}_{\ell}(\mathcal{S})$ the same way as during key generation. The signature is computed as

$$
\sigma=\left(\sigma_{1}, \ldots, \sigma_{\ell}\right)=\left(c^{b_{1}}\left(\mathbf{s k}_{1}, \mathbf{r}\right), \ldots, c^{b_{\ell}}\left(\mathbf{s k}_{\ell}, \mathbf{r}\right)\right)
$$

Verification Algorithm $\left(\mathrm{pk}^{\prime} \leftarrow \operatorname{WOTS} \cdot \mathrm{vf}(M, \sigma, \mathbf{r})\right)$ : On input of an $n$-bit message $M$, a signature $\sigma$, and bitmasks $\mathbf{r}$, the verification algorithm first computes the $b_{i}, 1 \leq i \leq \ell$ as described above. Then it returns:

$$
\mathrm{pk}^{\prime}=\left(\mathrm{pk}_{1}^{\prime}, \ldots, \mathrm{pk}_{\ell}^{\prime}\right)=\left(c^{w-1-b_{1}}\left(\sigma_{1}, \mathbf{r}_{b_{1}+1, w-1}\right), \ldots, c^{w-1-b_{\ell}}\left(\sigma_{\ell}, \mathbf{r}_{b_{\ell}+1, w-1}\right)\right) .
$$

A formally correct verification algorithm would compare $\mathrm{pk}^{\prime}$ to a given public key and output true on equality and false otherwise. In SPHINCS this comparison is delegated to the overall verification algorithm.

Binary Hash Trees. The central elements of our construction are full binary hash trees. We use the construction proposed in [18] shown in Figure 1.

In SPHINCS, a binary hash tree of height $h$ always has $2^{h}$ leaves which are $n$ bit strings $L_{i}, i \in\left[2^{h}-1\right]$. Each node $N_{i, j}$, for $0<j \leq h, 0 \leq i<2^{h-j}$, of the tree stores an $n$-bit string. To construct the tree, $h$ bit masks $\mathbf{Q}_{j} \in\{0,1\}^{2 n}$, $0<j \leq h$, are used. For the leaf nodes define $N_{i, 0}=L_{i}$. The values of the internal nodes $N_{i, j}$ are computed as

$$
N_{i, j}=\mathrm{H}\left(\left(N_{2 i, j-1} \| N_{2 i+1, j-1}\right) \oplus \mathbf{Q}_{j}\right) .
$$

We also denote the root as $\operatorname{Root}=N_{0, h}$.

An important notion is the authentication path $\operatorname{Auth}_{i}=\left(\mathrm{A}_{0}, \ldots, \mathrm{A}_{h-1}\right)$ of a leaf $L_{i}$ shown in Figure 2. Auth ${ }_{i}$ consists of all the sibling nodes of the nodes contained in the path from $L_{i}$ to the root. For a discussion on how to compute authentication paths, see Section 5. Given a leaf $L_{i}$ together with its authentication path Auth $_{i}$, the root of the tree can be computed using Algorithm 1. 
Input: Leaf index $i$, leaf $L_{i}$, authentication path $\mathrm{Auth}_{i}=\left(\mathrm{A}_{0}, \ldots, \mathrm{A}_{h-1}\right)$ for $L_{i}$. Output: Root node Root of the tree that contains $L_{i}$.

Set $P_{0} \leftarrow L_{i}$;

for $j \leftarrow 1$ up to $h$ do

$P_{j}= \begin{cases}\mathrm{H}\left(\left(P_{j-1} \| \mathrm{A}_{j-1}\right) \oplus \mathbf{Q}_{j}\right), & \text { if }\left\lfloor i / 2^{j-1}\right\rfloor \equiv 0 \bmod 2 ; \\ \mathrm{H}\left(\left(\mathrm{A}_{j-1} \| P_{j-1}\right) \oplus \mathbf{Q}_{j}\right), & \text { if }\left[i / 2^{j-1}\right\rfloor \equiv 1 \bmod 2 ;\end{cases}$

end

return $P_{h}$

Algorithm 1: Root Computation

L-Tree. In addition to the full binary trees above, we also use unbalanced binary trees called L-Trees as in [18]. These are exclusively used to hash WOTS $^{+}$public keys. The $\ell$ leaves of an L-Tree are the elements of a $\mathrm{WOTS}^{+}$public key and the tree is constructed as described above but with one difference: A left node that has no right sibling is lifted to a higher level of the L-Tree until it becomes the right sibling of another node. Apart from this the computations work the same as for binary trees. The L-Trees have height $\lceil\log \ell\rceil$ and hence need $\lceil\log \ell\rceil$ bitmasks.

\section{$2.1 \quad$ HORST}

HORST signs messages of length $m$ and uses parameters $k$ and $t=2^{\tau}$ with $k \tau=m$ (typical values as used in SPHINCS-256 are $t=2^{16}, k=32$ ). HORST improves HORS [34] using a binary hash-tree to reduce the public key size from $t n$ bits to $n$ bits $^{5}$ and the combined signature and public key size from $t n$ bits to $\left(k(\tau-x+1)+2^{x}\right) n$ bits for some $x \in \mathbb{N} \backslash\{0\}$. The value $x$ is determined based on $t$ and $k$ such that $k(\tau-x+1)+2^{x}$ is minimal. It might happen that the expression takes its minimum for two successive values. In this case the greater value is used. For SPHINCS-256 this results in $x=6$.

In contrast to a one-time signature scheme like WOTS, HORST can be used to sign more than one message with the same key pair. However, with each signature the security decreases. See Section 3 for more details. Like for WOTS ${ }^{+}$ our description includes pseudorandom key generation. We now describe the algorithms for HORST:

Key Generation Algorithm (pk $\leftarrow \operatorname{HORST} \cdot \operatorname{kg}(\mathcal{S}, \mathbf{Q}))$ : On input of seed $\mathcal{S} \in$ $\{0,1\}^{n}$ and bitmasks $\mathbf{Q} \in\{0,1\}^{2 n \times \log t}$ the key generation algorithm first computes the internal secret key sk $=\left(\mathrm{sk}_{1}, \ldots, \mathrm{sk}_{t}\right) \leftarrow \mathrm{G}_{t}(\mathcal{S})$. The leaves of the tree are computed as $L_{i}=\mathrm{F}\left(\mathbf{s k}_{i}\right)$ for $i \in[t-1]$ and the tree is constructed using bitmasks $\mathbf{Q}$. The public key pk is computed as the root node of a binary tree of height $\log t$.

${ }^{5}$ Here we assume that the used bitmasks are given as they are used for several key pairs. Otherwise, public key size is $(2 \tau+1) n$ bit including bitmasks, which is still less than $t n$ bits. 


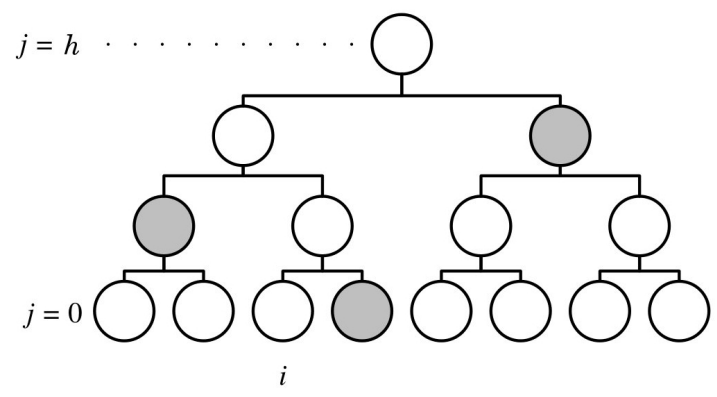

Fig. 2. The authentication path for leaf $L_{i}$

Signature Algorithm $((\sigma, \mathrm{pk}) \leftarrow \operatorname{HORST} \cdot \operatorname{sign}(M, \mathcal{S}, \mathbf{Q}))$ : On input of a message $M \in\{0,1\}^{m}$, seed $\mathcal{S} \in\{0,1\}^{n}$, and bitmasks $\mathbf{Q} \in\{0,1\}^{2 n \times \log t}$ first the internal secret key sk is computed as described above. Then, let $M=\left(M_{0}, \ldots, M_{k-1}\right)$ denote the $k$ numbers obtained by splitting $M$ into $k$ strings of length $\log t$ bits each and interpreting each as an unsigned integer. The signature $\sigma=$ $\left(\sigma_{0}, \ldots, \sigma_{k-1}, \sigma_{k}\right)$ consists of $k$ blocks $\sigma_{i}=\left(\right.$ sk $_{M_{i}}$, Auth $\left.M_{i}\right)$ for $i \in[k-1]$ containing the $M_{i}$ th secret key element and the lower $\tau-x$ elements of the authentication path of the corresponding leaf $\left(\mathrm{A}_{0}, \ldots, \mathrm{A}_{\tau-1-x}\right)$. The block $\sigma_{k}$ contains all the $2^{x}$ nodes of the binary tree on level $\tau-x\left(N_{0, \tau-x}, \ldots, N_{2^{x}-1, \tau-x}\right)$. In addition to the signature, HORST.sign also outputs the public key.

Verification Algorithm ( $\left.\mathrm{pk}^{\prime} \leftarrow \operatorname{HORST} \cdot \operatorname{vf}(M, \sigma, \mathbf{Q})\right)$ : On input of message $M \in$ $\{0,1\}^{m}$, a signature $\sigma$, and bitmasks $\mathbf{Q} \in\{0,1\}^{2 n \times \log t}$, the verification algorithm first computes the $M_{i}$, as described above. Then, for $i \in[k-1], y_{i}=\left\lfloor M_{i} / 2^{\tau}-x\right\rfloor$ it computes $N_{y_{i}, \tau-x}^{\prime}$ using Algorithm 1 with index $M_{i}, L_{M_{i}}=\mathrm{F}\left(\sigma_{i}^{1}\right)$, and Auth $_{M_{i}}=\sigma_{i}^{2}$. It then checks that $\forall i \in[k-1]: N_{y_{i}, \tau-x}^{\prime}=N_{y_{i}, \tau-x}$, i.e., that the computed nodes match those in $\sigma_{k}$. If all comparisons hold it uses $\sigma_{k}$ to compute and then return RooT $_{0}$, otherwise it returns fail.

Theoretical Performance. In the following we give rough theoretical performance values for HORST when used in a many-time signature scheme. We ignore the space needed for bitmasks, assuming they are provided. For runtimes we only count PRG calls and the number of hash evaluations without distinguishing the different hash functions.

Sizes: A HORST secret key consists of a single $n$ bit seed. The public key contains a single $n$ bit hash. A signature contains $k$ secret key elements and authentication paths of length $(\log t)-x$ (Recall $t=2^{\tau}$ is a power of two). In addition it contains $2^{x}$ nodes in $\sigma_{k}$, adding up to a total of $\left(k((\log t)-x+1)+2^{x}\right) n$ bits.

Runtimes: Key generation needs one evaluation of $\mathrm{G}_{t}$ and $t$ hashes to compute the leaf values and $t-1$ hashes to compute the public key, leading to a total of $2 t-1$. Signing takes the same time as we require the root is part of the output. Verification takes $k$ times one hash to compute a leaf value plus $(\log t)-x$ hashes 
to compute the node on level $(\log t)-x$. In addition, $2^{x}-1$ hashes are needed to compute the root from $\sigma_{k}$. Together these are $k((\log t)-x+1)+2^{x}-1$ hashes.

\section{$2.2 \quad$ SPHINCS}

Given all of the above we can finally describe the algorithms of the SPHINCS construction. A SPHINCS keypair completely defines a "virtual" structure which we explain first. SPHINCS works on a hyper-tree of height $h$ that consists of $d$ layers of trees of height $h / d$. Each of these trees looks as follows. The leaves of a tree are $2^{h / d}$ L-Tree root nodes that each compress the public key of a WOTS $^{+}$key pair. Hence, a tree can be viewed as a key pair that can be used to sign $2^{h / d}$ messages. The hyper-tree is structured into $d$ layers. On layer $d-1$ it has a single tree. On layer $d-2$ it has $2^{h / d}$ trees. The roots of these trees are signed using the WOTS ${ }^{+}$key pairs of the tree on layer $d-1$. In general, layer $i$ consists of $2^{(d-1-i)(h / d)}$ trees and the roots of these trees are signed using the $\mathrm{WOTS}^{+}$key pairs of the trees on layer $i+1$. Finally, on layer 0 each WOTS $^{+}$key pair is used to sign a HORST public key. We talk about a "virtual" structure as all values within are determined choosing a seed and the bitmasks, and as the full structure is never computed. The seed is part of the secret key and used for pseudorandom key generation. To support easier understanding, Figure 3 shows the virtual structure of a SPHINCS signature, i.e. of one path inside the hyper-tree. It contains $d$ trees $\operatorname{TREE}_{i} i \in[d-1]$ (each consisting of a binary hash tree that authenticates the root nodes of $2^{h / d}$ L-Trees which in turn each have the public key nodes of one $\mathrm{WOTS}^{+}$keypair as leaves). Each tree authenticates the tree below using a $\mathrm{WOTS}^{+}$signature $\sigma_{W, i}$. The only exception is $\mathrm{TREE}_{0}$ which authenticates a HORST public key using a $\mathrm{WOTS}^{+}$signature. Finally, the HORST key pair is used to sign the message. Which trees inside the hyper-tree are used (which in turn determines the WOTS $^{+}$key pairs used for the signature) and which HORST key pair is determined by the pseudorandomly generated index not shown here.

We use a simple addressing scheme for pseudorandom key generation. An address is a bit string of length $a=\lceil\log (d+1)\rceil+(d-1)(h / d)+(h / d)=$ $\lceil\log (d+1)\rceil+h$. The address of a WOTS $^{+}$key pair is obtained by encoding the layer of the tree it belongs to as a $\log (d+1)$-bit string (using $d-1$ for the top layer with a single tree). Then, appending the index of the tree in the layer encoded as a $(d-1)(h / d)$-bit string (we number the trees from left to right, starting with 0 for the left-most tree). Finally, appending the index of the $\mathrm{WOTS}^{+}$key pair within the tree encoded as a $(h / d)$-bit string (again numbering from left to right, starting with 0 ). The address of the HORST key pair is obtained using the address of the WOTS $^{+}$key pair used to sign its public key and placing $d$ as the layer value in the address string, encoded as $\lceil\log (d+1)\rceil$ bit string. To give an example: In SPHINCS-256, an address needs 64 bits.

Key Generation Algorithm $\left((\mathrm{SK}, \mathrm{PK}) \leftarrow \operatorname{kg}\left(1^{n}\right)\right)$ : The key generation algorithm first samples two secret key values $\left(\mathrm{SK}_{1}, \mathrm{SK}_{2}\right) \in\{0,1\}^{n} \times\{0,1\}^{n}$. The value $\mathrm{SK}_{1}$ is used for pseudorandom key generation. The value $\mathrm{SK}_{2}$ is used to generate an 


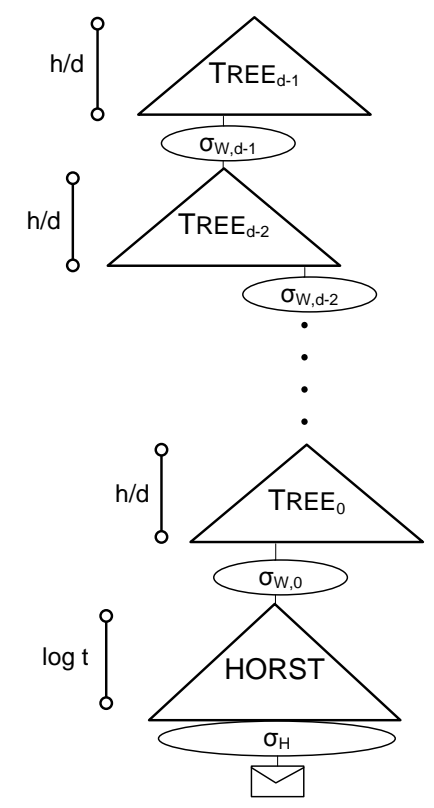

Fig. 3. Virtual structure of a SPHINCS signature

unpredictable index in sign and pseudorandom values to randomize the message hash in sign. Also, $p$ uniformly random $n$-bit values $\mathbf{Q} \stackrel{\$}{\leftarrow}\{0,1\}^{p \times n}$ are sampled as bitmasks where $p=\max \{w-1,2(h+\lceil\log \ell\rceil), 2 \log t\}$. These bitmasks are used for all $\mathrm{WOTS}^{+}$and HORST instances as well as for the trees. In the following we use $\mathbf{Q}_{\text {WOTS }}$ for the first $w-1$ bitmasks (of length $n$ ) in $\mathbf{Q}, \mathbf{Q}_{\text {HORST }}$ for the first $2 \log t, \mathbf{Q}_{L-\text { Tree }}$ for the first $2\lceil\log \ell\rceil$, and $\mathbf{Q}_{\text {Tree }}$ for the $2 h$ strings of length $n$ in $\mathbf{Q}$ that follow $\mathbf{Q}_{L-T r e e}$.

The remaining part of $\mathrm{kg}$ consists of generating the root node of the tree on layer $d-1$. Towards this end the WOTS $^{+}$key pairs for the single tree on layer $d-1$ are generated. The seed for the key pair with address $A=(d-1\|0\| i)$ where $i \in\left[2^{h / d}-1\right]$ is computed as $\mathcal{S}_{A} \leftarrow \mathcal{F}_{a}\left(A, \mathrm{SK}_{1}\right)$, evaluating the PRF on input $A$ with key $\mathrm{SK}_{1}$. In general, the seed for a WOTS $^{+}$key pair with address $A$ is computed as $\mathcal{S}_{A} \leftarrow \mathcal{F}_{a}\left(A, \mathrm{SK}_{1}\right)$ and we will assume from now on that these seeds are known to any algorithm that knows $\mathrm{SK}_{1}$. The $\mathrm{WOTS}^{+}$public key is computed as $\mathrm{pk}_{A} \leftarrow$ WOTS.kg $\left(\mathcal{S}_{A}, \mathbf{Q}_{\text {WOTS }^{+}}\right)$. The $i$ th leaf $L_{i}$ of the tree is the root of an L-Tree that compresses $\mathrm{pk}_{A}$ using bit masks $\mathbf{Q}_{L-\text { Tree}}$. Finally, a binary hash tree is built using the constructed leaves and its root node becomes $\mathrm{PK}_{1}$.

The SPHINCS secret key is $\mathrm{SK}=\left(\mathrm{SK}_{1}, \mathrm{SK}_{2}, \mathbf{Q}\right)$, the public key is $\mathrm{PK}=$ $\left(\mathrm{PK}_{1}, \mathbf{Q}\right)$. kg returns the key pair $\left(\left(\mathrm{SK}_{1}, \mathrm{SK}_{2}, \mathbf{Q}\right),\left(\mathrm{PK}_{1}, \mathbf{Q}\right)\right)$.

Signature Algorithm $(\Sigma \leftarrow \operatorname{sign}(M, \mathrm{SK}))$ : On input of a message $M \in\{0,1\}^{*}$ and secret key $\mathrm{SK}=\left(\mathrm{SK}_{1}, \mathrm{SK}_{2}, \mathbf{Q}\right)$, sign computes a randomized message digest $D \in$ $\{0,1\}^{m}$ : First, a pseudorandom $R=\left(R_{1}, R_{2}\right) \in\{0,1\}^{n} \times\{0,1\}^{n}$ is computed as $R \leftarrow \mathcal{F}\left(M, \mathrm{SK}_{2}\right)$. Then, $D \leftarrow \mathcal{H}\left(R_{1}, M\right)$ is computed as the randomized hash of $M$ using the first $n$ bits of $R$ as randomness. The latter $n$ bits of $R$ are used to select a HORST keypair, computing an $h$ bit index $i \leftarrow \operatorname{CHOP}\left(R_{2}, h\right)$ as the first 
$h$ bits of $R_{2}$. Note, that signing is deterministic, i.e. we need no real randomness as all required 'randomness' is pseudorandomly generated using PRF $\mathcal{F}$.

Given index $i$, the HORST key pair with address $A_{\text {HORST }}=(d \| i(0,(d-$ 1) $h / d) \| i((d-1) h / d, h / d))$ is used to sign the message digest $D$, i.e., the first $(d-1) h / d$ bits of $i$ are used as tree index and the remaining bits for the index within the tree. The HORST signature and public key $\left(\sigma_{\mathrm{H}}, \mathrm{pk}_{\mathrm{H}}\right) \leftarrow\left(D, \mathcal{S}_{A_{\text {HORST }}}\right.$, $\left.\mathbf{Q}_{\text {HORST }}\right)$ are computed using the HORST bitmasks and the seed $\mathcal{S}_{A_{\text {HORST }}} \leftarrow$ $\mathcal{F}_{a}\left(A_{\mathrm{HORST}}, \mathrm{SK}_{1}\right)$.

The SPHINCS signature $\Sigma=\left(i, R_{1}, \sigma_{\mathrm{H}}, \sigma_{\mathrm{W}, 0}\right.$, Auth $_{A_{0}}, \ldots, \sigma_{\mathrm{W}, d-1}$, Auth $\left._{A_{d-1}}\right)$ contains besides index $i$, randomness $R_{1}$ and HORST signature $\sigma_{\mathrm{H}}$ also one WOTS $^{+}$signature and one authentication path $\sigma_{\mathrm{W}, i}$, Auth $_{A_{i}}, i \in[d-2]$ per layer. These are computed as follows: The WOTS $^{+}$key pair with address $A_{0}$ is used to sign $\mathrm{pk}_{\mathrm{H}}$, where $A_{0}$ is the address obtained taking $A_{\mathrm{HORST}}$ and setting the first $\lceil\log (d+1)\rceil$ bits to zero. This is done running $\sigma_{\mathrm{W}, 1} \leftarrow\left(\mathrm{pk}_{\mathrm{H}}, \mathcal{S}_{A_{0}}, \mathbf{Q}_{\mathrm{WOTS}^{+}}\right)$ using the $\mathrm{WOTS}^{+}$bitmasks. Then the authentication path $\operatorname{Auth}_{i((d-1) h / d, h / d))}$ of the used WOTS $^{+}$key pair is computed. Next, the WOTS $^{+}$public key pk ${ }_{\mathrm{W}, 0}$ is computed running $\mathrm{pk}_{\mathrm{W}, 0} \leftarrow \operatorname{WOTS} \cdot \mathrm{vf}\left(\mathrm{pk}_{\mathrm{H}}, \sigma_{\mathrm{W}, 0}, \mathbf{Q}_{\mathrm{WOTS}^{+}}\right)$. The root node RooT $_{0}$ of the tree is computed by first compressing $\mathrm{pk}_{\mathrm{W}, 0}$ using an L-Tree. Then Algorithm 1 is applied using the index of the WOTS $^{+}$key pair within the tree, the root of the L-Tree and $\operatorname{Auth}_{i((d-1) h / d, h / d))}$.

This procedure gets repeated for layers 1 to $d-1$ with the following two differences. On layer $1 \leq j<d$, WOTS ${ }^{+}$is used to sign $\operatorname{RooT}_{j-1}$, the root computed at the end of the previous iteration. The address of the WOTS $^{+}$key pair used on layer $j$ is computed as $A_{j}=(j\|i(0,(d-1-j) h / d)\| i((d-1-$ $j) h / d, h / d)$ ), i.e. on each layer the last $(h / d)$ bits of the tree address become the new leaf address and the remaining bits of the former tree address become the new tree address.

Finally, sign outputs $\Sigma=\left(i, R_{1}, \sigma_{\mathrm{H}}, \sigma_{\mathrm{W}, 0}\right.$, Auth $_{A_{0}}, \ldots, \sigma_{\mathrm{W}, d-1}$, Auth $\left._{A_{d-1}}\right)$.

Verification Algorithm $(b \leftarrow \operatorname{vf}(M, \Sigma, \mathrm{PK}))$ : On input of a message $M \in\{0,1\}^{*}$, a signature $\Sigma$, and a public key PK, the algorithm computes the message digest $D \leftarrow \mathcal{H}\left(R_{1}, M\right)$ using the randomness $R_{1}$ contained in the signature. The message digest $D$ and the HORST bitmasks $\mathbf{Q}_{\text {HORST }}$ from PK are used to compute the HORST public key $\mathrm{pk}_{\mathrm{H}} \leftarrow \operatorname{HORST.vf}\left(D, \sigma_{\mathrm{H}}, \mathbf{Q}_{\mathrm{HORST}}\right)$ from the HORST signature. If HORST.vf returns fail, verification returns false. The HORST public key in turn is used together with the $\mathrm{WOTS}^{+}$bit masks and the WOTS ${ }^{+}$ signature to compute the first WOTS ${ }^{+}$public key $\mathrm{pk}_{\mathrm{W}, 0} \leftarrow$ WOTS.vf $\left(\mathrm{pk}_{\mathrm{H}}, \sigma_{\mathrm{W}, 0}\right.$, $\left.\mathbf{Q}_{\mathrm{WOTS}^{+}}\right)$. An L-Tree is used to compute $L_{i((d-1) h / d, h / d)}$, the leaf corresponding to $\mathrm{pk}_{\mathrm{W}, 0}$. Then, the root $\mathrm{RoOT}_{0}$ of the respective tree is computed using Algorithm 1 with index $i((d-1) h / d, h / d)$, leaf $L_{i((d-1) h / d, h / d)}$ and authentication path Auth $_{0}$.

Then, this procedure gets repeated for layers 1 to $d-1$ with the following two differences. First, on layer $1 \leq j<d$ the root of the previously processed tree $\operatorname{RoOT}_{j-1}$ is used to compute the WOTS ${ }^{+}$public key $\mathrm{pk}_{\mathrm{W}, j}$. Second, the leaf computed from $\mathrm{pk}_{\mathrm{W}, j}$ using an L-Tree is $L_{i((d-1-j) h / d, h / d)}$, i.e., the index of 
the leaf within the tree can be computed cutting off the last $j(h / d)$ bits of $i$ and then using the last $(h / d)$ bits of the resulting bit string.

The result of the final repetition on layer $d-1$ is a value $\operatorname{Root}_{d-1}$ for the root node of the single tree on the top layer. This value is compared to the first element of the public key, i.e., $\mathrm{PK}_{1} \stackrel{?}{=} \operatorname{RoOT}_{d-1}$. If the comparison holds, vf returns true, otherwise it returns false.

Theoretical Performance. In the following we give rough theoretical performance values. We count runtimes counting the number of PRF, PRG and hash evaluations without distinguishing the different PRFs, PRGs, and hashes.

Sizes: A SPHINCS secret key consists of two $n$ bit seeds and the $p=\max \{w-$ $1,2(h+\lceil\log \ell\rceil), 2 \log t\} n$ bit bitmasks, summing up to $(2+p) n$ bits. The public key contains a single $n$ bit hash and the bitmasks: $(1+p) n$ bits. A signature contains one $h$ bit index and $n$ bits of randomness. Moreover, it contains a HORST signature $\left(\left(k((\log t)-x+1)+2^{x}\right) n\right.$ bits $), d$ WOTS signatures ( $\ell n$ bits each), and a total of $h$ authentication path nodes ( $n$ bits each). This gives a signature size of $\left(\left(k((\log t)-x+1)+2^{x}\right)+d \ell+h+1\right) n+h$ bits.

Runtimes: SPHINCS key generation consists of building the top tree. This takes for leaf generation $2^{h / d}$ times the following: One PRF call, one PRG call, one WOTS $^{+}$key generation ( $\ell w$ hashes), and one L-Tree $(\ell-1$ hashes). Building the tree adds another $2^{h / d}-1$ hashes. Together these are $2^{h / d} \mathrm{PRF}$ and PRG calls and $(\ell(w+1)) 2^{h / d}-1$ hashes. Signing requires one PRF call to generate the index and the randomness for the message hash as well as the message hash itself. Then one PRF call to generate a HORST seed and a HORST signature. In addition, $d$ trees have to be built, adding $d$ times the time for key generation. The WOTS $^{+}$signatures can be extracted while running WOTS ${ }^{+}$key generation, hence they add no extra cost. This sums up to $d 2^{h / d}+2$ PRF calls, $d 2^{h / d}+1$ PRG calls, and $2 t+d\left((\ell(w+1)) 2^{h / d}-1\right)$ hashes. Finally, verification needs the message hash, one HORST verification, and $d$ times a WOTS $^{+}$verification ( $<\ell w$ hashes), computing an L-Tree, and $h / d-1$ hashes to compute the root. This leads to a total of $k((\log t)-x+1)+2^{x}+d(\ell(w+1)-2)+h$ hashes.

\section{Security Analysis}

We now discuss the security of SPHINCS. We first give a reduction from standard hash function properties. Afterwards we discuss the best generic attacks on these properties using quantum computers. Definitions of the used properties can be found in Appendix A. For our security analysis we group the message hash and the mapping used within HORST to a function $\mathcal{H}_{k, t}$ that maps bit strings of arbitrary length to a subset of $\{0, \ldots, t-1\}$ with at most $k$ elements.

\subsection{Security Reduction}

We will now prove our main theorem which states that SPHINCS is secure as long as the used function (families) provide certain standard security proper- 
ties. These properties are fulfilled by secure cryptographic hash functions, even against quantum attacks. For the exact statement in the proof we use the notion of insecurity functions. An insecurity function $\operatorname{InSec}^{p}(s ; t, q)$ describes the maximum success probability of any adversary against property $p$ of primitive $s$, running in time $\leq t$, and (if given oracle access, e.g. to a signing oracle) making no more than $q$ queries. To avoid the non-constructive high-probability attacks discussed in [11], we measure time with the $A T$ metric rather than the RAM metric. Properties are one-wayness (OW), second-preimage resistance (SPR), undetectability (UD), secure pseudorandom generator (PRG), secure pseudorandom function family (PRF), and $\gamma$-subset resilience $(\gamma$-sr).

Theorem 1. SPHINCS is existentially unforgeable under $q_{s}$-adaptive chosen message attacks if

- F is a second-preimage resistant, undetectable one-way function,

- $\mathrm{H}$ is a second-preimage resistant hash function,

- $\mathrm{G}_{\lambda}$ is a secure pseudorandom generator for values $\lambda \in\{\ell, t\}$,

$-\mathcal{F}_{\lambda}$ is a pseudorandom function family for $\lambda=a$,

- $\mathcal{F}$ is a pseudorandom function family, and

- for the subset-resilience of $\mathcal{H}_{k, t}$ it holds that

$$
\sum_{\gamma=1}^{\infty} \min \left\{2^{\gamma\left(\log q_{s}-h\right)+h}, 1\right\} \cdot \operatorname{Succ}_{\mathcal{H}_{k, t}^{\gamma-s r}}(\mathcal{A})=\operatorname{negl}(n)
$$

for any probabilistic polynomial-time adversary $\mathcal{A}$, where $\operatorname{Succ}_{\mathcal{H}_{k, t}}^{\gamma-s r}(\mathcal{A})$ denotes the success probability of $\mathcal{A}$ against the $\gamma$-subset resilience of $\mathcal{H}_{k, t}$.

More specifically, the insecurity function $\operatorname{InSec}{ }^{\mathrm{EU}-\mathrm{CMA}}\left(S P H I N C S ; \xi, q_{s}\right)$ describing the maximum success probability of all adversaries against the existential unforgeability under $q_{s}$-adaptive chosen message attacks, running in time $\leq \xi$, is bounded by

$$
\begin{aligned}
\operatorname{InSec}^{\mathrm{EU}-\mathrm{CMA}}\left(S P H I N C S ; \xi, q_{s}\right) \\
\quad \leq \operatorname{InSec}^{\mathrm{PRF}}\left(\mathcal{F} ; \xi, q_{s}\right)+\operatorname{InSec}^{\mathrm{PRF}}\left(\mathcal{F}_{a} ; \xi, \#_{f t s}+\#_{o t s}\right) \\
\quad+\#_{o t s} \cdot \operatorname{InSec}{ }^{\mathrm{PRG}}\left(\mathrm{G}_{\ell} ; \xi\right)+\#_{f t s} \cdot \operatorname{InSec}^{\mathrm{PRG}}\left(\mathrm{G}_{t} ; \xi\right) \\
\quad+\#_{t r e e} \cdot 2^{h / d+\lceil\log \ell\rceil} \cdot \operatorname{InSec}^{\mathrm{SPR}}(\mathrm{H} ; \xi) \\
\quad+\#_{o t s} \cdot\left(\ell w^{2} \cdot \operatorname{InSec}^{\mathrm{UD}}(\mathrm{F} ; \xi)+\ell w \cdot \operatorname{InSec}^{\mathrm{OW}}(\mathrm{F} ; \xi)+\ell w^{2} \cdot \operatorname{InSec}^{\mathrm{SPR}}(\mathrm{F} ; \xi)\right) \\
\quad+\#_{f t s} \cdot 2 t \cdot \operatorname{InSec}{ }^{\mathrm{SPR}}(\mathrm{H} ; \xi)+\#_{f t s} \cdot t \cdot \operatorname{InSec}^{\mathrm{OW}}(\mathrm{F} ; \xi) \\
\left.\quad+\sum_{\gamma=1}^{\infty} \min \left\{2^{\gamma\left(\log q_{s}-h\right)+h}, 1\right\} \cdot \operatorname{InSec} \operatorname{Sesr}^{\gamma-\mathcal{H}_{k, t}} ; \xi\right),
\end{aligned}
$$

where $\#_{\text {ots }}=\min \left\{\sum_{i=1}^{d} 2^{i h / d}, d q_{s}\right\}$ denotes the maximum number of $\mathrm{WOTS}^{+}$ key pairs, $\#_{f t s}=\min \left\{2^{h}, q_{s}\right\}$ denotes the maximum number of HORST key pairs, and $\#_{\text {tree }}=\min \left\{\sum_{i=1}^{d-1} 2^{i h / d},(d-1) q_{s}\right\}$ denotes the maximum number of subtrees used answering $q_{s}$ signature queries. 
Before we give the proof, note that although there is a bunch of factors within the exact security statement, the reduction is tight. All the factors are constant / independent of the security parameter. They arise as all the primitives are used many times. E.g., the pseudorandom generator $\mathrm{G}_{\ell}$ is used for every WOTS ${ }^{+}$ key pair and an adversary can forge a signature if it can distinguish the output for one out of the $\#_{\text {ots }}$ applications of $\mathrm{G}_{\ell}$ from a random bit string. Similar explanations exist for the other factors.

Proof. In the following we first show that the success probability of any probabilistic polynomial-time adversary $\mathcal{A}$ that attacks the EU-CMA security of SPHINCS is negligible in the security parameter. Afterwards, we analyze the exact success probability of $\mathcal{A}$ and show that it indeed fulfills the claimed bound. First consider the following six games:

Game 1 is the original EU-CMA game against SPHINCS.

Game 2 differs from Game 1 in that the value $R$ used to randomize the message hash and to choose the index $i$ is chosen uniformly at random instead of using $\mathcal{F}$.

Game 3 is similar to Game 2 but this time all used WOTS ${ }^{+}$and HORST seeds are generated uniformly at random and stored in some list for reuse instead of generating them using $\mathcal{F}_{a}$.

Game 4 is similar to Game 3 but this time no pseudorandom key generation is used inside $\mathrm{WOTS}^{+}$. Instead, all $\mathrm{WOTS}^{+}$secret key elements are generated uniformly at random and stored in some list for reuse.

Game 5 is similar to Game 4 but this time no pseudorandom key generation is used at all. Instead, also all HORST secret key elements are generated uniformly at random and stored in some list for reuse.

The difference in the success probability of $\mathcal{A}$ between playing Game 1 and Game 2 must be negligible. Otherwise we could use $\mathcal{A}$ as an distinguisher against the pseudorandomness of $\mathcal{F}$. Similarly, the difference in the success probability of $\mathcal{A}$ between playing Game 2 and Game 3 must be negligible. Otherwise, we could use $\mathcal{A}$ as an distinguisher against the pseudorandomness of $\mathcal{F}_{a}$. Also the difference in the success probability of $\mathcal{A}$ between playing Game 3 and Game 4 and playing Game 4 and Game 5 must be negligible. Otherwise, $\mathcal{A}$ could be used to distinguish the outputs of the $\mathrm{PRG} \mathrm{G}_{\ell}\left(\right.$ resp. $\mathrm{G}_{t}$ ) from uniformly random bit strings.

It remains to limit the success probability of $\mathcal{A}$ running in Game 5. Assume that $\mathcal{A}$ makes $q_{s}$ queries to the signing oracle before outputting a valid forgery

$$
M^{*}, \Sigma^{*}=\left(i^{*}, R^{*}, \sigma_{\mathrm{H}}^{*}, \sigma_{\mathrm{W}, 0}^{*}, \text { Auth }_{A_{0}}^{*}, \ldots, \sigma_{\mathrm{W}, d-1}^{*}, \text { Auth }_{A_{d-1}}^{*}\right) .
$$

The index $i^{*}$ was used to sign at least one of the query messages with overwhelming probability. Otherwise, $\mathcal{A}$ could be turned into a (second-)preimage finder for $\mathrm{H}$ that succeeds with non-negligible probability. Hence, we assume from now on $i^{*}$ was used before. While running $\operatorname{vf}\left(M^{*}, \Sigma^{*}, \mathrm{PK}\right)$ we can extract the computed HORST public key $\mathrm{pk}_{\mathrm{H}}^{*}$ as well as the computed WOTS ${ }^{+}$public keys $\mathrm{pk}_{\mathrm{W}, j}^{*}$ and 
the root nodes of the trees containing these $\mathrm{WOTS}^{+}$public keys $\mathrm{RoOT}_{j}^{*}$ for all levels $j \in[d-1]$. In addition, we compute the respective values $\mathrm{pk}_{\mathrm{H}}, \mathrm{pk}_{\mathrm{W}, j}$ and $\mathrm{RoOT}_{j}$ using the list of secret key elements. All required elements must be contained in the lists as $i^{*}$ was used before.

Next we compare these values in reverse order of computation, i.e., starting with $\mathrm{pk}_{\mathrm{W}, d-1} \stackrel{?}{=} \mathrm{pk}_{\mathrm{W}, d-1}^{*}$, then $\operatorname{RooT}_{d-2} \stackrel{?}{=} \operatorname{RoOT}_{d-2}^{*}$, and so forth. Then one of the following four mutually exclusive cases must appear:

Case 1: The first occurrence of a difference happens for a $\mathrm{WOTS}^{+}$public key. As shown in [18] this can only happen with negligible probability. Otherwise, we can use $\mathcal{A}$ to compute second-preimages for $\mathrm{H}$ with non-negligible success probability.

Case 2: The first difference occurs for two root nodes $\operatorname{RooT}_{j} \neq \mathrm{RoOT}_{j}^{*}$. This implies a forgery for the $\mathrm{WOTS}^{+}$key pair used to sign Root ${ }_{j}$. As shown in [26] this can only happen with negligible advantage. Otherwise, we could use $\mathcal{A}$ to either break the one-wayness, the second-preimage resistance, or the undetectability of $\mathrm{F}$ with non-negligible success probability.

Case 3: The first spotted difference is two different HORST public keys. As for Case 2, this implies a $\mathrm{WOTS}^{+}$forgery and can hence only appear with negligible probability.

Case 4: All the public keys and root nodes are equal, i.e. no difference occurs.

We excluded all cases but Case 4 which we analyze now. The analysis consists of a sequence of mutually exclusive cases. Recall that the secret key elements for this HORST key pair are already fixed and contained in the secret value list as $i^{*}$ was used in the query phase. First, we compare the values of all leaf nodes that can be derived from $\sigma_{\mathrm{H}}^{*}$ with the respective values derived from the list entries. These are the hashes of the secret key elements in the signature and the authentication path nodes for level 0 . The case that there exists a difference can only appear with negligible probability, as otherwise $\mathcal{A}$ could be used to compute second-preimages for $\mathrm{H}$ with non-negligible probability following the proof in [18]. Hence, we assume from now on all of these are equal.

Second, the indices of the secret key values contained in $\sigma_{\mathrm{H}}^{*}$ have either all been published as parts of query signatures or at least one index has not been published before. The latter case can only appear with negligible probability. Otherwise, $\mathcal{A}$ could be turned into a preimage finder for $\mathrm{F}$ that has non-negligible success probability. Finally, we can limit the probability that all indices have been published as parts of previous signatures.

Recall, when computing the signatures on the query messages, the indices were chosen uniformly at random. Hence, the probability that a given index reoccurs $\gamma$ times, i.e., is used for $\gamma$ signatures, is equal to the probability of the event $C\left(2^{h}, q_{s}, \gamma\right)$ that after $q_{s}$ samples from a set of size $2^{h}$ at least one value was sampled $\gamma$ times. This probability can in turn be bound by

$$
\operatorname{Pr}\left[C\left(2^{h}, q_{s}, \gamma\right)\right] \leq \frac{1}{2^{h(\gamma-1)}}\left(\begin{array}{c}
q_{s} \\
\gamma
\end{array}\right) \leq \frac{q_{s}^{\gamma}}{2^{h(\gamma-1)}}=2^{\gamma\left(\log q_{s}-h\right)+h}
$$


as shown in [36]. Using Equation (1), the probability for this last case can be written as

$$
\sum_{\gamma=1}^{\infty} \min \left\{2^{\gamma\left(\log q_{s}-h\right)+h}, 1\right\} \cdot \operatorname{Succ}_{\mathcal{H}_{k, t}-\mathrm{sr}}(\mathcal{A})
$$

i.e. the sum over the probabilities that there exists at least one index that was used $\gamma$ times multiplied by $\mathcal{A}$ 's success probability against the $\gamma$-subset resilience of $\mathcal{H}_{k, t}$. This sum is negligible per assumption. Hence the success probability of $\mathcal{A}$ is negligible which concludes the asymptotic proof.

Probabilities. Now we take a look at the exact success probability $\epsilon=$

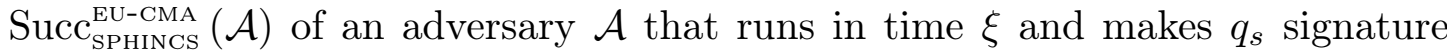
queries. Per definition, $\mathcal{A}$ 's probability of winning Game 1 is $\epsilon$. In what follows let $\#_{\text {ots }}=\min \left\{\sum_{i=1}^{d} 2^{i h / d}, d q_{s}\right\}$ denote the maximum number of $\mathrm{WOTS}^{+}$ key pairs, $\#_{\text {fts }}=\min \left\{2^{h}, q_{s}\right\}$ the maximum number of HORST key pairs, and $\#_{\text {tree }}=\min \left\{\sum_{i=1}^{d-1} 2^{i h / d},(d-1) q_{s}\right\}$ the maximum number of subtrees used while answering signature queries. Now, from the definition of the insecurity functions we get that the differences in the success probabilities of $\mathcal{A}$ playing two neighboring games from the above series of games are bounded by $\operatorname{InSec}{ }^{\mathrm{PRF}}\left(\mathcal{F} ; \xi, q_{s}\right), \operatorname{InSec}{ }^{\mathrm{PRF}}\left(\mathcal{F}_{a} ; \xi, \#_{\text {fts }}+\#_{\text {ots }}\right), \#_{\text {ots }} \cdot \operatorname{InSec}^{\mathrm{PRG}}\left(\mathrm{G}_{\ell} ; \xi\right), \#_{\text {fts }}$. InSec ${ }^{\text {PRG }}\left(\mathrm{G}_{t} ; \xi\right)$, respectively. Hence, $\epsilon$ is bounded by $\mathcal{A}$ 's probability of winning Game 5 plus the sum of the above bounds.

It remains to limit $\mathcal{A}$ 's success probability in Game 5. A more detailed analysis shows that the case that $i^{*}$ was not used before is also covered by the following cases. (The reason is that at some point the path from the message to the root must meet a path which was used in the response to a query before.) So we only have to consider the four cases. The probability that $\mathcal{A}$ succeeds with a Case 1 forgery is limited by $\#_{t r e e} \cdot 2^{h / d+\lceil\log \ell\rceil} \cdot \operatorname{InSec}{ }^{\mathrm{SPR}}(\mathrm{H} ; \xi)$. This bound can be obtained by first guessing a tree and then following the proof in [18]. The combined probability that $\mathcal{A}$ succeeds with a Case 2 or Case 3 forgery is limited by $\#_{o t s} \cdot \operatorname{InSec}^{\mathrm{EU}-\mathrm{CMA}}\left(\mathrm{WOTS}^{+} ; t, 1\right) \leq \#_{\text {ots }} \cdot\left(\ell w^{2} \cdot \operatorname{InSec}^{\mathrm{UD}}(\mathrm{F} ; \xi)+\ell w \cdot\right.$ $\left.\operatorname{InSec}^{\mathrm{OW}}(\mathrm{F} ; \xi)+\ell w^{2} \cdot \operatorname{InSec}^{\mathrm{SPR}}(\mathrm{F} ; \xi)\right)$. Similarly to the last case, this bound can be obtained by first guessing the $\mathrm{WOTS}^{+}$key pair $\mathcal{A}$ will forge a signature for and then following the proof from $[26]^{6}$.

Case 4 consists of another three mutually exclusive cases. The probability that $\mathcal{A}$ succeeds by inserting new leaves into the HORST tree can be bounded by $\#_{f t s} \cdot 2 t \cdot \operatorname{InSec}^{\mathrm{SPR}}(\mathrm{H} ; \xi)$. This can be seen, first guessing the HORST key pair and then following again the proof in [18]. The probability that $\mathcal{A}$ succeeds by providing a valid value for an index not included in previous signatures can be bounded by $\#_{f t s} \cdot t \cdot \operatorname{InSec}{ }^{\mathrm{OW}}(\mathrm{F} ; \xi)$. This can be shown, first guessing the HORST key pair and afterwards, guessing the index. Finally, the probability that $\mathcal{A}$ succeeds finding a message for which it already knows the values to be opened from previous signatures can be bounded by $\sum_{\gamma=1}^{\infty} \min \left\{2^{\gamma\left(\log q_{s}-h\right)+h}, 1\right\} \cdot \operatorname{InSec}^{\gamma-\operatorname{sr}}\left(\mathcal{H}_{k, t} ; \xi\right)$.

\footnotetext{
${ }^{6}$ The used bound is actually an improved bound from [25].
} 
As the three cases are mutually exclusive, the probability that $\mathcal{A}$ succeeds with a Case 4 forgery is bound by the sum of the three probabilities above. Similarly, the probability of $\mathcal{A}$ winning Game 5 is bound by the sum of the probabilities of $\mathcal{A}$ succeeding in Case 1 - 4. This finally leads the claimed bound.

\subsection{Generic attacks}

As a complement to the above reduction, we now analyze the concrete complexity of various attacks, both pre-quantum and post-quantum. Recall that $\mathcal{H}_{k, t}(R, M)$ applied to message $M \in\{0,1\}^{*}$ and randomness $R \in\{0,1\}^{n}$ works as follows. First, the message digest is computed as $M^{\prime}=\mathcal{H}(R, M) \in\{0,1\}^{m}$. Then, $M^{\prime}$ is split into $k$ bit strings, each of length $\log t$. Finally, each of these bit strings is interpreted as an unsigned integer. Thus, the output of $\mathcal{H}_{k, t}$ is an ordered subset of $k$ values out of the set $[t-1]$ (possibly with repetitions).

Subset-Resilience. The main attack vector against SPHINCS is targeting subset-resilience. The obvious first attack is to simply replace $(R, M)$ in a valid signature with $\left(R^{\prime}, M^{\prime}\right)$, hoping that $\mathcal{H}_{k, t}(R, M)=\mathcal{H}_{k, t}\left(R^{\prime}, M^{\prime}\right)$. This violates strong unforgeability if $(R, M) \neq\left(R^{\prime}, M^{\prime}\right)$, and it violates existential unforgeability if $M \neq M^{\prime}$. Finding a second preimage of $(R, M)$ under $\mathcal{H}_{k, t}$ costs $2^{m}$ pre-quantum but only $2^{m / 2}$ post-quantum (Grover's algorithm). To reach success probability $p$ takes time $\sqrt{p} 2^{m / 2}$.

The attacker does succeed in reusing $\mathcal{H}_{k, t}(R, M)$ if $\mathcal{H}_{k, t}\left(R^{\prime}, M^{\prime}\right)$ contains the same indices as $\mathcal{H}_{k, t}(R, M)$, because then he can permute the HORST signature for $(R, M)$ accordingly to obtain the HORST signature for $\left(R^{\prime}, M^{\prime}\right)$. If $k^{2}$ is considerably smaller than $t$ then the $k$ indices in a hash are unlikely to contain any collisions, so there are about $2^{m} / k$ ! equivalence classes of hashes under permutations. It is easy to map each hash to a numerical representative of its equivalence class, effectively reducing the pre-quantum second-preimage cost from $2^{m}$ to $2^{m} / k$ !, and the post-quantum second-preimage cost from $2^{m / 2}$ to $\sqrt{2^{m} / k !}$.

More generally, when $\gamma$ valid signatures use the same HORST key, the attacker can mix and match the HORST signatures. All the attacker needs is to break $\gamma$-subset-resilience: i.e., find $\mathcal{H}_{k, t}\left(R^{\prime}, M^{\prime}\right)$ so that the set of $k$ indices in it is a subset of the union of the indices in the $\gamma$ valid signatures. The union has size about $\gamma k$ (at most $\gamma k$, and usually close to $\gamma k$ if $\gamma k$ is not very large compared to $t$ ), so a uniform random number has probability about $\gamma k / t$ of being in the union, and if the $k$ indices were independent uniform random numbers then they would have probability about $(\gamma k)^{k} / t^{k}$ of all being in the union. The expected cost of a pre-quantum attack is about $t^{k} /(\gamma k)^{k}$, and the expected cost of a post-quantum attack is about $t^{k / 2} /(\gamma k)^{k / 2}$.

Of course, this attack cannot start unless the signer in fact produced $\gamma$ valid signatures using the same HORST key. After a total of $q$ signatures, the probability of any particular HORST key being used exactly $\gamma$ times is exactly $\left(\begin{array}{l}q \\ \gamma\end{array}\right)\left(1-1 / 2^{h}\right)^{q-\gamma}\left(1 / 2^{h}\right)^{\gamma}$. This probability is bounded above by $\left(q / 2^{h}\right)^{\gamma}$ in the 
above proof; a much tighter approximation is $\left(q / 2^{h}\right)^{\gamma} \exp \left(-q / 2^{h}\right) / \gamma$ !. If the ratio $\rho=q / 2^{h}$ is significantly smaller than 1 then there will be approximately $q$ keys used once, approximately $\rho q / 2$ keys used twice, approximately $\rho^{2} q / 6$ keys used three times, and so on. The chance that some key will be used $\gamma$ times, for $\gamma=h / \log (1 / \rho)+\delta$, is approximately $\rho^{\delta} / \gamma$ !.

For example, consider $t=2^{16}, k=32, h=60$, and $q=2^{50}$. There is a noticeable chance, approximately $2^{-9.5}$, that some HORST key is used 6 times. For $\gamma=6$ the expected cost of a pre-quantum attack is $2^{269}$ and the expected cost of a post-quantum attack is $2^{134}$. Increasing $\gamma$ to 9 reduces the post-quantum cost below $2^{128}$, specifically to $2^{125.3}$, but the probability of a HORST key being used 9 times is below $2^{-48}$. Increasing $\gamma$ to $10,11,12,13,14,15$ reduces the cost to $2^{122.8}, 2^{120.6}, 2^{118.6}, 2^{116.8}, 2^{115.1}, 2^{113.5}$ respectively, but reduces the probability to $2^{-61}, 2^{-75}, 2^{-88}, 2^{-102}, 2^{-116}, 2^{-130}$ respectively.

Security degrades as $q$ grows closer to $2^{h}$. For example, for $q=2^{60}$ the attacker finds $\gamma=26$ with probability above $2^{-30}$, and then a post-quantum attack costs only about $2^{100}$. Of course, the signer is in control of the number of messages signed: for example, even if the signer's key is shared across enough devices to continuously sign $2^{20}$ messages per second, signing $2^{50}$ messages would take more than 30 years.

One-Wayness. The attacker can also try to work backwards from a hash output to an $n$-bit hash input that was not revealed by the signer (or a $n$-bit half of a $2 n$-bit hash input where the other half was revealed by the signer). If the hash inputs were independent uniform random $n$-bit strings then this would be a standard preimage problem; generic pre-quantum preimage search costs $2^{n}$, and generic post-quantum preimage search (again Grover) costs $2^{n / 2}$.

The attacker can also merge preimage searches for $n$-bit-to- $n$-bit hashes. (For $2 n$-bit-to- $n$-bit hashes the known $n$ input bits have negligible chance of repeating.) For pre-quantum attacks the cost of generic $T$-target preimage attacks is well known to drop by a factor of $T$; here $T$ is bounded by approximately $2^{h}$ (the exact bound depends on $q$ ), for a total attack cost of approximately $2^{n-h}$. For post-quantum attacks, it is well known that $2^{n / 2} / \sqrt{T}$ quantum queries are necessary and sufficient for generic $T$-target preimage attacks (assuming $T<2^{n / 3}$ ), but there is overhead beyond the queries. An analysis of this overhead by Bernstein [8] concludes that all known post-quantum collision-finding algorithms cost at least $2^{n / 2}$, implying that the post-quantum cost of multi-target preimage attacks is also $2^{n / 2}$. For example, for $n=256$ and $T=2^{56}$ the best post-quantum attacks use only $2^{100}$ queries but still cost $2^{128}$.

Second-Preimage Resistance. As for the message hash, finding a second preimage of either a message $M \in\{0,1\}^{n}$ under $\mathrm{F}$ or a message $\mathcal{M} \in\{0,1\}^{2 n}$ under $\mathrm{H}$ costs $2^{n}$ pre-quantum and $2^{n / 2}$ post-quantum (Grover's algorithm).

PRF, PRG, and Undetectability. The hash inputs are actually obtained from a chain of PRF outputs, PRG outputs, and lower-level hash outputs. The attacker can try to find patterns in these inputs, for example by guessing the PRF key, the PRG seed, or an input to F that ends up at a target value after 
a number of rounds of the chaining function. All generic attacks again cost $2^{n}$ pre-quantum and $2^{n / 2}$ post-quantum.

\section{SPHINCS-256}

In addition to the general construction of SPHINCS, we propose a specific instantiation called SPHINCS-256. The parameters, functions, and resulting key and signature sizes of SPHINCS-256 are summarized in Table 1. This section describes how these parameters and functions were chosen.

Parameters. The parameters for SPHINCS-256 were selected with two goals in mind: (1) long-term $2^{128}$ security against attackers with access to quantum computers; (2) a good tradeoff between speed and signature size. The first goal determined the security parameter $n=256$, which in turn determined the name SPHINCS-256. Optimizing the remaining parameters required deciding on the relative importance of speed and signature size. After searching a large parameter space we settled on the parameters $m=512, h=60, d=12, w=16, t=2^{16}$, and $k=32$, implying $\ell=67, x=6$, and $a=64$. These choices are small enough and fast enough for a wide range of applications. Of course, one can also define different SPHINCS instantiations, changing the remaining parameters in favor of either speed or signature size.

Security of SPHINCS-256. SPHINCS-256 uses $n=256, m=512, h=$ $60, d=12, w=16, t=2^{16}, k=32$ as parameters. Hence, considering attackers that have access to a large scale quantum computer this means the following. Assuming the best attacks against the used hash functions are generic attacks as described in the last section, $\mathcal{H}_{k, t}$ provides security above $2^{128}$ regarding subsetresilience, $\mathrm{F}$ and $\mathrm{H}$ provide $2^{128}$ security against preimage, second-preimage and in case of F undetectability attacks. Similarly, the used PRFs and PRGs provide security $2^{128}$. Summing up, SPHINCS-256 provides $2^{128}$ security against post-quantum attackers under the assumptions above.

Fast Fixed-Size Hashing. The primary cost metric in the literature on cryptographic hash functions, for example in the recently concluded SHA-3 competition, is performance for long inputs. However, what is most important for SPHINCS and hash-based signatures in general is performance for short inputs. The hashing in SPHINCS consists primarily of applying $\mathrm{F}$ to $n$-bit inputs and secondarily of applying $\mathrm{H}$ to $2 n$-bit inputs.

Short-input performance was emphasized in a recent MAC/PRF design [1] from Aumasson and Bernstein. We propose short-input performance as a similarly interesting target for hash-function designers.

Designing a new hash function is not within the scope of this paper: we limit ourselves to evaluating the short-input performance of previously designed components that appear to have received adequate study. Below we explain our selection of specific functions $\mathrm{F}:\{0,1\}^{n} \rightarrow\{0,1\}^{n}$ and $\mathrm{H}:\{0,1\}^{2 n} \rightarrow\{0,1\}^{n}$ for $n=256$. 


\begin{tabular}{|l|r|l|}
\hline Parameter & Value & Meaning \\
\hline$n$ & 256 & bitlength of hashes in HORST and WOTS \\
\hline$m$ & 512 & bitlength of the message hash \\
\hline$h$ & 60 & height of the hyper-tree \\
\hline$d$ & 12 & layers of the hyper-tree \\
\hline$w$ & 16 & Winternitz parameter used for WOTS signatures \\
\hline$t$ & $2^{16}$ & number of secret-key elements of HORST \\
\hline$k$ & 32 & number of revealed secret-key elements per HORST sig. \\
\hline \hline \multicolumn{3}{|c|}{ Functions } \\
\hline Hash $\mathcal{H}:$ & $\mathcal{H}(R, M)=$ BLAKE-512 $(R \| M)$ \\
\hline PRF $\mathcal{F}_{a}:$ & $\mathcal{F}_{a}(A, K)=$ BLAKE-256 $(K \| A)$ \\
\hline PRF $\mathcal{F}:$ & $\mathcal{F}(M, K)=$ BLAKE-512(K\|M) \\
\hline PRG $\mathrm{G}_{\lambda}:$ & $\mathrm{G}_{\lambda}(\mathrm{SEED})=$ ChaCha12 ${ }_{\mathrm{SEED}}(0)_{0, \ldots, \lambda-1}$ \\
\hline Hash F: & $\mathrm{F}\left(M_{1}\right)=$ CHOP $\left(\pi_{\mathrm{ChaCha}}\left(M_{1} \| C\right), 256\right)$ \\
\hline Hash H: & $\mathrm{H}\left(M_{1} \| M_{2}\right)=$ CHOP $\left(\pi_{\mathrm{ChaCha}}\left(\pi_{\mathrm{ChaCha}}\left(M_{1} \| C\right) \oplus\left(M_{2} \| 0^{256}\right)\right), 256\right)$ \\
\hline \hline \multicolumn{4}{|l|}{ Sizes } \\
\hline Signature size: & 41000 bytes \\
\hline Public-key size: 1056 bytes \\
\hline Private-key size: 1088 bytes \\
\hline
\end{tabular}

Table 1. SPHINCS-256 parameters and functions for the 128-bit post-quantum security level and resulting signature and key sizes.

Review of Permutation-Based Cryptography. Rivest suggested strengthening the DES cipher by "whitening" the input and output: i.e., encrypting a block $M$ under key $\left(K, K_{1}, K_{2}\right)$ as $E_{K}\left(M \oplus K_{1}\right) \oplus K_{2}$, where $E_{K}$ means DES using key $K$. Even and Mansour [21] suggested eliminating the original key $K$ : i.e., encrypting a block $M$ under key $\left(K_{1}, K_{2}\right)$ as $E\left(M \oplus K_{1}\right) \oplus K_{2}$, where $E$ is an unkeyed public permutation. Kilian and Rogaway [28, Section 4] suggested taking $K_{1}=K_{2}$.

Combining all of these suggestions means encrypting $M$ under key $K$ as $E(M \oplus K) \oplus K$; see, e.g., [29], [7], and [20]. Trivial 0-padding or, more generally, padding with a constant allows $M$ and $K$ to be shorter than the block length of $E$ : for example, the "Salsa20" cipher from [7] actually produces $E(K, M, C)+$ $(K, M, C)$, where $C$ is a constant. The PRF security of Salsa20 is tightly equivalent to the PRF security of $E(K, M, C)+(K, 0,0)$, which in turn implies the PRF security of the "HSalsa20" stream cipher [9] obtained by truncating $E(K, M, C)$.

Bertoni, Daemen, Peeters, and Van Assche [12] proposed building cryptographic hash functions from unkeyed permutations, and later proposed a specific "Keccak" hash function. The "sponge" construction used in [12], and in Keccak, hashes a $(b-c)$-bit message $K_{1}$ to a $(b-c)$-bit truncation of $E\left(K_{1}, C\right)$, where $C$ is a $c$-bit constant; hashes a $2(b-c)$-bit message $\left(K_{1}, K_{2}\right)$ to a $(b-c)$-bit truncation of $E\left(E\left(K_{1}, C\right) \oplus\left(K_{2}, 0\right)\right)$; etc. Sponges have been reused in many subsequent designs and studied in many papers. We ended up selecting the sponge structure for both $\mathrm{F}$ and $\mathrm{H}$. 
Note that the single-block hash here, a truncation of $E\left(K_{1}, C\right)$, is the same as an encryption of a constant nonce using a truncated- $E\left(K_{1}, M, C\right)$ cipher. Of course, there is no logical connection between the PRF security of this cipher and (e.g.) second-preimage resistance, but designers use the same techniques to build $E$ for either context: consider, for example, the reuse of the Salsa20 permutation in the "Rumba20" [5] compression function, the reuse of a tweaked version of the "ChaCha20" permutation [6] in the "BLAKE" and "BLAKE2" [4] hash functions, and the reuse of the Keccak permutation in the "Keyak" [14] authenticated-encryption scheme.

Many other hash-function designs use input blocks as cipher keys, but in most cases the underlying ciphers use complicated "key schedules" rather than wrapping simple key addition around an unkeyed permutation. Both [7] and [13] state reasons to believe that unkeyed permutations provide the best performancesecurity tradeoff. Performance obviously played a large role in the selection of Salsa20/12 (Salsa20 reduced to 12 rounds) for the eSTREAM portfolio, the deployment of ChaCha20 in TLS [31], and the selection of Keccak as SHA-3. We did not find any non-permutation-based hash-function software competitive in performance with the permutation that we selected.

Choice of Permutation for $\boldsymbol{n}=256$. A sponge function using a $b$-bit permutation $E$ and a $c$-bit "capacity" takes $b-c$ bits in each input block and produces $b-c$ bits of output. We require $b-c \geq 256$ so that a single call to $E$ hashes 256 bits to 256 bits (and two calls to $E$ hash 512 bits to 256 bits). The attacker can compute preimages by guessing the $c$ missing bits and applying $E^{-1}$, so we also require $c \geq 256$.

We considered using the Keccak permutation, which has $b=1600$, but this is overkill: it takes as long to hash a 256-bit block as it does to hash a 1000-bit block. There is a scaled-down version of Keccak with $b=800$, but this is not part of SHA-3, and we do not know how intensively it has been analyzed.

After considering various other permutations we settled on ChaCha, which has $b=512$. ChaCha is a slightly modified version of Salsa, advertising faster diffusion and at the same time better performance. The best key-recovery attacks known are from Aumasson, Fischer, Khazaei, Meier, and Rechberger [2] and are slightly faster than $2^{256}$ operations against 8 rounds of Salsa and 7 rounds of ChaCha, supporting the security advertisement. The eSTREAM portfolio recommends 12 rounds of Salsa20 as having a "comfortable margin for security" so we selected 12 rounds of ChaCha (ChaCha12). The Salsa and ChaCha permutations are not designed to simulate ideal permutations: they are designed to simulate ideal permutations with certain symmetries, i.e., ideal permutations of the orbits of the state space under these symmetries. The Salsa and ChaCha stream ciphers add their inputs to only part of the block and specify the rest of the block as asymmetric constants, guaranteeing that different inputs lie in different orbits. For the same reason we specify an asymmetric constant for $C$.

Specifically, let $\pi_{\text {ChaCha }}:\{0,1\}^{512} \rightarrow\{0,1\}^{512}$ denote the ChaCha12 permutation, let $C$ be the bytes of the ASCII representation of "expand 32-byte to 64-byte state!" and let $\operatorname{CHOp}(M, i)$ be the function that returns the first $i$ bits 
of the string $M$. Then we define

$$
\begin{aligned}
\mathrm{F}\left(M_{1}\right) & =\operatorname{CHOp}\left(\pi_{\text {ChaCha }}\left(M_{1} \| C\right), 256\right), \text { and } \\
\mathrm{H}\left(M_{1} \| M_{2}\right) & =\operatorname{CHOP}\left(\pi_{\text {ChaCha }}\left(\pi_{\text {ChaCha }}\left(M_{1} \| C\right) \oplus\left(M_{2} \| 0^{256}\right)\right), 256\right) .
\end{aligned}
$$

for any 256-bit strings $M_{1}, M_{2}$.

Other functions. We also use ChaCha12 directly for the PRG $\mathrm{G}_{\lambda}$. Specifically, we define $\mathrm{G}_{\lambda}(\mathrm{SEED})=$ ChaCha12 $2_{\mathrm{SeED}}(0)_{0, \ldots, \lambda-1}$, i.e., we run ChaCha12 with key SEED and initialization vector 0 and take the first $\lambda$ output bits.

For message hashing we use BLAKE, whose security was extensively studied as part of the SHA-3 competition. We also use BLAKE for the $n$-bit-output PRF and for the $2 n$-bit-output PRF: We define $\mathcal{H}(R, M)=\operatorname{BLAKE}-512(R \| M)$; $\mathcal{F}_{a}(A, K)=$ BLAKE-256 $(K \| A)$; and $\mathcal{F}(M, K)=\operatorname{BLAKE}-512(K \| M)$.

\section{Fast software implementation}

The fastest arithmetic units of most modern microprocessors are vector units. Instead of performing a certain arithmetic operation on scalar inputs, they perform the same operation in parallel on multiple values kept in vector registers. Not surprisingly, many speed records for cryptographic algorithms are held by implementations that make efficient use of these vector units. Also not surprisingly, many modern cryptographic primitives are designed with vectorizability in mind. In this section we describe how to efficiently implement SPHINCS-256 using vector instructions, more specifically the AVX2 vector instructions in Intel Haswell processors. All cycle counts reported in this section are measured on one core of an Intel Xeon E3-1275 CPU running at 3.5 GHz. We followed the standard practice of turning off Turbo Boost and hyperthreading for our benchmarks.

The AVX2 Instruction Set. The Advanced Vector Extensions (AVX) were introduced by Intel in 2011 with the Sandy Bridge microarchitecture. The extensions feature 16 vector registers of size 256 bits. In AVX, those registers can only be used as vectors of 8 single-precision floating-point values or vectors of 4 double-precision floating-point values. This functionality was extended in AVX2, introduced with the Haswell microarchitecture, to also support arithmetic on 256 -bit vectors of integers of various sizes. We use these AVX2 instructions for 8 -way parallel computations on 32-bit integers.

Vectorizing Hash Computations. The two low-level operations in SPHINCS that account for most of the computations are the fixed-input-size hash functions $\mathrm{F}$ and $\mathrm{H}$. The SPHINCS-256 instantiation of $\mathrm{F}$ and $\mathrm{H}$ internally uses the ChaCha permutation. We now discuss vectorized computation of this permutation.

An obvious approach is to use the same parallelism exploited in [3, Section 3.1.3], which notes that the core operations in ChaCha and BLAKE "can be computed in four parallel branches". Most high-speed implementations of BLAKE use this internal 4-way parallelism for vector computations. 
However, it is much more efficient to vectorize across multiple independent computations of $\mathrm{F}$ or $\mathrm{H}$. The most obvious reason is that the ChaCha permutation operates on 32-bit integers which means that 4-way-parallel computation can only make use of half of the 256-bit AVX vector registers. A second reason is that internal vectorization of ChaCha requires relatively frequent shuffling of values in vector registers. Those shuffles do not incur a serious performance penalty, but they are noticeable. A third reason is that vectorization is not the only way that modern microprocessors exploit parallelism. Instruction-level parallelism is used on pipelined processors to hide latencies and superscalar CPUs can even execute multiple independent instruction in the same cycle. A non-vectorized implementation of ChaCha has 4-way instruction-level parallelism which makes very good use of pipelining and superscalar execution. A vectorized implementation of ChaCha has almost no instruction-level parallelism and suffers from serious instruction-latency penalties.

Our 8-way parallel implementation of $\mathrm{F}$ takes 420 cycles to hash 8 independent 256-bit inputs to 8256 -bit outputs. Our 8 -way parallel implementation of $\mathrm{H}$ takes 836 cycles to hash 8 independent 512-bit inputs to 8256 -bit outputs. These speeds assume that the inputs are interleaved in memory. Interleaving and de-interleaving data means transposing an $8 \times 8$ 32-bit-word matrix.

This vectorization across 8 simultaneous hash computations is suitable for the two main components in the SPHINCS signature generation, namely HORST signing and WOTS authentication-path computations, as described below. The same approach also generalizes to other instantiations of $\mathrm{F}$ and $\mathrm{H}$, although for some functions it is more natural to use 64-bit words.

HORST Signing. The first step in HORST signature generation is to expand the secret seed into a stream of $t \cdot n=16777216$ bits (or $2 \mathrm{MB}$ ). This pseudorandom stream forms the $2^{16}$ secret keys of HORST. We use ChaCha12 for this seed expansion, more specifically Andrew Moon's implementation of ChaCha12, which SUPERCOP identifies as the fastest implementation for Haswell CPUs. The seed expansion costs about 1814424 cycles.

The costly part of HORST signing is to first evaluate $\mathrm{F}\left(\mathrm{sk}_{i}\right)$ for $i=0, \ldots, t-$ 1 , and then build the binary hash tree on top of the $\mathrm{F}\left(\mathrm{sk}_{i}\right)$ and extract nodes which are required for the 32 authentication paths. SPHINCS-256 uses $t=2^{16}$ so we need a total of 65536 evaluations of $\mathrm{F}$ and 65535 evaluations of $\mathrm{H}$. A streamlined vectorized implementation treats the HORST binary tree up to level 13 (the level with 8 nodes) as 8 independent sub-trees and vectorizes computations across these sub-trees. Data needs to be interleaved only once at the beginning (the HORST secret keys $\mathbf{s k}_{i}$ ) and de-interleaved at the very end (the 8 nodes on level 13 of the HORST tree). All computations in between are streamlined 8way parallel computations of $\mathrm{F}$ and $\mathrm{H}$ on interleaved data. The final tree hashing from level 13 to the HORST root at level 16 needs only 7 evaluations of H. This is a negligible cost, even when using a slower non-vectorized implementation of $\mathrm{H}$.

Note that, strictly speaking, we do not have to interleave input at all; we can simply treat the $2 \mathrm{MB}$ output of ChaCha12 as already interleaved random data. 
However, this complicates compatible non-vectorized or differently vectorized implementations on other platforms.

WOTS Authentication Paths. Computing a WOTS authentication path consists of 32 WOTS key generations, each followed by an L-Tree computation. This produces 32 WOTS public keys, which form the leaves of a binary tree. Computing this binary tree and extracting the nodes required for the authentication path finishes this computation. The costly part of this operation is the computation of 32 WOTS public keys $(32 \cdot 15 \cdot 67=32160$ evaluations of $\mathrm{F}$ ) and of 32 L-Tree computations $(32 \cdot 66=2112$ evaluations of $\mathrm{H})$. For comparison, the binary tree at the end costs only 31 computations of $\mathrm{H}$. Efficient vectorization parallelizes across 8 independent WOTS public-key computations with subsequent L-Tree computations. Data needs to be interleaved only once at the very beginning (the WOTS secret key) and de-interleaved once at the very end (the roots of the L-Trees). Again, all computations in between are streamlined 8-way parallel computations of $\mathrm{F}$ and $\mathrm{H}$ on interleaved data.

SPHINCS Signing Performance. Our software still uses some more transpositions of data than the almost perfectly streamlined implementation described above. With these transpositions and some additional overhead to xor hash inputs with masks, update pointers and indices etc., HORST signing takes 15033564 cycles. The lower bound from 65536 evaluations of $\mathrm{F}$ and 65535 evaluations of $\mathrm{H}$ is 10289047 cycles. The computation of one WOTS authentication path takes 2586784 cycles. The lower bound from 32160 evaluations of $\mathrm{F}$ and 2143 evaluations of $\mathrm{H}$ is 1912343 cycles. The complete SPHINCS-256 signing takes 51636372 cycles; almost all of these cycles are explained by one HORST signature and 12 WOTS authentication paths.

SPHINCS Key Generation and Verification. The by far most costly operation in SPHINCS is signing so we focused our optimization efforts on this operation. Some easily vectorizable parts of key generation and verification also use our high-speed 8-way vectorized implementations of $\mathrm{F}$ and $\mathrm{H}$, but other parts still use relatively slow non-vectorized versions based on the ChaCha12 reference implementation in eBACS [10]. Our implementation of key generation takes 3237260 cycles. Our implementation of signature verification takes 1451004 cycles.

RAM Usage and Size. Our implementation is optimized for speed on large Intel processors where size and memory usage are typically only a minor concern. Consequently, we did not optimize for those parameters. For example, we keep the complete HORST tree in memory and then extract the hashes that are needed in the 32 authentication paths. This approach keeps the software simple, but if we wanted to save memory, we would instead use treehash [32] to construct the tree and extract and store required authentication-path entries on the fly. Although the software is not optimized for memory usage, we do not need any dynamic memory allocations; all temporary data fits into the Linux default stack limit of $8 \mathrm{MB}$. The size of the complete signing software, including BLAKE for message hashing, is $104 \mathrm{~KB}$. 
Acknowledgement Thanks to Christian Rechberger and Andrew Miller for helpful discussions on the topic.

\section{References}

1. Jean-Philippe Aumasson and Daniel J. Bernstein. SipHash: A fast short-input PRF. In Steven D. Galbraith and Mridul Nandi, editors, Progress in Cryptology INDOCRYPT 2012, volume 7668 of LNCS, pages 489-508. Springer, 2012.

2. Jean-Philippe Aumasson, Simon Fischer, Shahram Khazaei, Willi Meier, and Christian Rechberger. New features of Latin dances: Analysis of Salsa, ChaCha, and Rumba. In Kaisa Nyberg, editor, Fast Software Encryption, volume 5086 of LNCS, pages 470-488. Springer, 2008.

3. Jean-Philippe Aumasson, Luca Henzen, Willi Meier, and Raphael C.-W. Phan. SHA-3 proposal BLAKE. Submission to NIST, 2008. http://131002.net/blake/ blake.pdf.

4. Jean-Philippe Aumasson, Samuel Neves, Zooko Wilcox-O'Hearn, and Christian Winnerlein. BLAKE2: Simpler, smaller, fast as MD5. In Michael J. Jacobson Jr., Michael E. Locasto, Payman Mohassel, and Reihaneh Safavi-Naini, editors, Applied Cryptography and Network Security, volume 7954 of LNCS, pages 119135. Springer, 2013.

5. Daniel J. Bernstein. What output size resists collisions in a xor of independent expansions? ECRYPT Hash Workshop, 2007.

6. Daniel J. Bernstein. ChaCha, a variant of Salsa20. SASC 2008: The State of the Art of Stream Ciphers, 2008.

7. Daniel J. Bernstein. The Salsa20 family of stream ciphers. In Matthew J. B. Robshaw and Olivier Billet, editors, New Stream Cipher Designs - The eSTREAM Finalists, volume 4986 of LNCS, pages 84-97. Springer, 2008.

8. Daniel J. Bernstein. Cost analysis of hash collisions: Will quantum computers make SHARCS obsolete? Workshop Record of SHARCS'09: Special-purpose Hardware for Attacking Cryptographic Systems, 2009.

9. Daniel J. Bernstein. Extending the Salsa20 nonce. Symmetric Key Encryption Workshop 2011, 2011.

10. Daniel J. Bernstein and Tanja Lange. eBACS: ECRYPT benchmarking of cryptographic systems. http://bench.cr.yp.to (accessed 2014-05-25).

11. Daniel J. Bernstein and Tanja Lange. Non-uniform cracks in the concrete: the power of free precomputation. In Kazue Sako and Palash Sarkar, editors, $A d$ vances in Cryptology - ASIACRYPT 2013, volume 8270 of LNCS, pages 321-340. Springer, 2013.

12. Guido Bertoni, Joan Daemen, Michaël Peeters, and Gilles Van Assche. Sponge functions. ECRYPT Hash Workshop, 2007.

13. Guido Bertoni, Joan Daemen, Michaël Peeters, and Gilles Van Assche. The road from Panama to Keccak via RadioGatún. Dagstuhl Seminar Proceedings, 2009.

14. Guido Bertoni, Joan Daemen, Michaël Peeters, Gilles Van Assche, and Ronny Van Keer. CAESAR submission: Keyak v1, 2014.

15. Johannes Buchmann, Erik Dahmen, and Andreas Hülsing. XMSS - a practical forward secure signature scheme based on minimal security assumptions. In Bo-Yin Yang, editor, Post-Quantum Cryptography, volume 7071 of LNCS, pages 117-129. Springer, 2011. 
16. Johannes Buchmann, Erik Dahmen, Elena Klintsevich, Katsuyuki Okeya, and Camille Vuillaume. Merkle signatures with virtually unlimited signature capacity. In Jonathan Katz and Moti Yung, editors, Applied Cryptography and Network Security, volume 4521 of LNCS, pages 31-45. Springer, 2007.

17. Johannes Buchmann, L. C. Coronado García, Erik Dahmen, Martin Döring, and Elena Klintsevich. CMSS - an improved Merkle signature scheme. In Rana Barua and Tanja Lange, editors, Progress in Cryptology - INDOCRYPT 2006, volume 4329 of LNCS, pages 349-363. Springer, 2006.

18. Erik Dahmen, Katsuyuki Okeya, Tsuyoshi Takagi, and Camille Vuillaume. Digital signatures out of second-preimage resistant hash functions. In Johannes Buchmann and Jintai Ding, editors, Post-Quantum Cryptography, volume 5299 of LNCS, pages 109-123. Springer, 2008.

19. Léo Ducas, Alain Durmus, Tancrède Lepoint, and Vadim Lyubashevsky. Lattice signatures and bimodal gaussians. In Ran Canetti and Juan A. Garay, editors, Advances in Cryptology - CRYPTO 2013, volume 8042 of LNCS, pages 40-56. Springer, 2013.

20. Orr Dunkelman, Nathan Keller, and Adi Shamir. Minimalism in cryptography: The Even-Mansour scheme revisited. In David Pointcheval and Thomas Johansson, editors, Advances in Cryptology - EUROCRYPT 2012, volume 7237 of LNCS, pages 336-354. Springer, 2012.

21. Shimon Even and Yishay Mansour. A construction of a cipher from a single pseudorandom permutation. Journal of Cryptology, 10(3):151-161, 1997.

22. Oded Goldreich. Two remarks concerning the goldwasser-micali-rivest signature scheme. In Andrew M. Odlyzko, editor, Advances in Cryptology - CRYPTO '86, volume 263 of $L N C S$, pages 104-110. Springer, 1987.

23. Oded Goldreich. Foundations of Cryptography: Volume 2, Basic Applications. Cambridge University Press, Cambridge, UK, 2004.

24. Shafi Goldwasser, Silvio Micali, and Ronald L. Rivest. A digital signature scheme secure against adaptive chosen-message attacks. SIAM Journal on Computing, 17(2):281-308, 1988.

25. Andreas Hülsing. Practical Forward Secure Signatures using Minimal Security Assumptions. PhD thesis, TU Darmstadt, 2013.

26. Andreas Hülsing. W-OTS + - shorter signatures for hash-based signature schemes. In Amr Youssef, Abderrahmane Nitaj, and Aboul-Ella Hassanien, editors, Progress in Cryptology - AFRICACRYPT 2013, volume 7918 of LNCS, pages 173-188. Springer, 2013.

27. Andreas Hülsing, Lea Rausch, and Johannes Buchmann. Optimal parameters for $\mathrm{XMSS}^{M T}$. In Alfredo Cuzzocrea, Christian Kittl, Dimitris E. Simos, Edgar Weippl, and Lida Xu, editors, Security Engineering and Intelligence Informatics, volume 8128 of LNCS, pages 194-208. Springer, 2013.

28. Joe Kilian and Phillip Rogaway. How to protect DES against exhaustive key search (an analysis of DESX). Journal of Cryptology, 14(1):17-35, 2001.

29. Kaoru Kurosawa. Power of a public random permutation and its application to authenticated-encryption. Cryptology ePrint Archive, Report 2002/127, 2002.

30. Leslie Lamport. Constructing digital signatures from a one way function. Technical Report SRI-CSL-98, SRI International Computer Science Laboratory, 1979.

31. Adam Langley. TLS symmetric crypto, 2014. https://www.imperialviolet.org/ 2014/02/27/tlssymmetriccrypto.html.

32. Ralph Merkle. A certified digital signature. In Gilles Brassard, editor, Advances in Cryptology - CRYPTO '89, volume 435 of LNCS, pages 218-238. Springer, 1990. 
33. Josef Pieprzyk, Huaxiong Wang, and Chaoping Xing. Multiple-time signature schemes against adaptive chosen message attacks. In Mitsuru Matsui and Robert Zuccherato, editors, Selected Areas in Cryptography, volume 3006 of LNCS, pages 88-100. Springer, 2004.

34. Leonid Reyzin and Natan Reyzin. Better than BiBa: Short one-time signatures with fast signing and verifying. In Lynn Batten and Jennifer Seberry, editors, Information Security and Privacy 2002, volume 2384 of LNCS, pages 1-47. Springer, 2002.

35. Fang Song. A note on quantum security for post-quantum cryptography. In Michele Mosca, editor, Post-Quantum Cryptography, volume 8772 of LNCS, pages 246-265. Springer, 2014.

36. Kazuhiro Suzuki, Dongvu Tonien, Kaoru Kurosawa, and Koji Toyota. Birthday paradox for multi-collisions. In Min Rhee and Byoungcheon Lee, editors, Information Security and Cryptology - ICISC 2006, volume 4296 of LNCS, pages 29-40. Springer, 2006.

\section{A Security Properties}

In this appendix we give the basic definitions for security properties we use.

Existential Unforgeability under Adaptive Chosen Message Attacks.

The standard security notion for digital signature schemes is existential unforgeability under adaptive chosen message attacks (EU-CMA) [24] which is defined using the following experiment. By $\operatorname{Dss}\left(1^{n}\right)$ we denote a signature scheme with security parameter $n$.

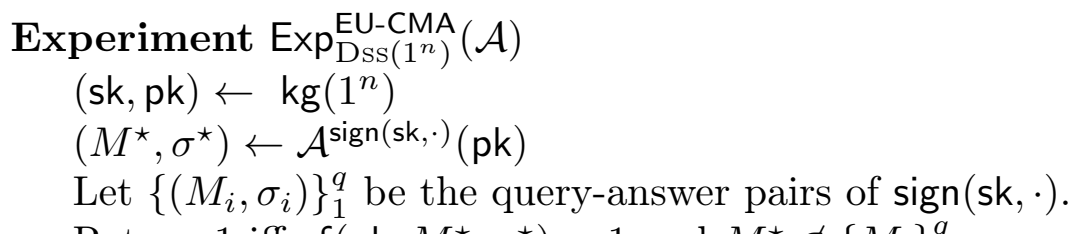

Return 1 iff $\operatorname{vf}\left(\mathrm{pk}, M^{\star}, \sigma^{\star}\right)=1$ and $M^{\star} \notin\left\{M_{i}\right\}_{1}^{q}$.

A signature scheme is called existentially unforgeable under a $q$ adaptive chosen message attack if any PPT adversary making at most $q$ queries, has only negligible success probability in winning the above game.

An EU-CMA secure one-time signature scheme (OTS) is a signature scheme that is existentially unforgeable under a 1-adaptively chosen message attack.

Hash Function Families. We now provide definitions of the security properties of hash function families that we use, namely one-wayness, second-preimage resistance, undetectability and pseudorandomness. In the following let $n \in \mathbb{N}$ be the security parameter, $m, k=\operatorname{poly}(n), \mathcal{H}_{n}=\left\{\mathrm{H}_{K}:\{0,1\}^{m} \rightarrow\{0,1\}^{n} \mid K \in\right.$ $\left.\{0,1\}^{k}\right\}$ a family of functions. (In the description of SPHINCS we actually omit the key $K$ in many cases for readability.)

We define the security properties in terms of the success probability of an adversary $\mathcal{A}$ against the respective property. A function family $\mathcal{H}_{n}$ is said to provide a property if the success probability of any probabilistic polynomialtime adversary against this property is negligible. We begin with the success 
probability of an adversary $\mathcal{A}$ against the one-wayness (ow) of a function family $\mathcal{H}_{n}$.

$$
\begin{gathered}
\operatorname{Succ}_{\mathcal{H}_{n}}^{\text {ow }}(\mathcal{A})=\operatorname{Pr}\left[K \stackrel{\$}{\leftarrow}\{0,1\}^{k} ; M \stackrel{\$}{\leftarrow}\{0,1\}^{m}, Y \leftarrow \mathrm{H}_{K}(M),\right. \\
\left.M^{\prime} \leftarrow \mathcal{A}(K, Y): Y=\mathrm{H}_{K}\left(M^{\prime}\right)\right] .
\end{gathered}
$$

We next define the success probability of an adversary $\mathcal{A}$ against second-preimage resistance (SPR).

$$
\begin{aligned}
\operatorname{Succ}_{\mathcal{H}_{n}}^{\mathrm{SPR}}(\mathcal{A})= & \operatorname{Pr}\left[K \stackrel{\$}{\leftarrow}\{0,1\}^{k} ; M \stackrel{\$}{\leftarrow}\{0,1\}^{m}, M^{\prime} \leftarrow \mathcal{A}(K, M):\right. \\
& \left.\left(M \neq M^{\prime}\right) \wedge\left(\mathrm{H}_{K}(M)=\mathrm{H}_{K}\left(M^{\prime}\right)\right)\right] .
\end{aligned}
$$

To define undetectability, assume the following two distributions over $\{0,1\}^{n} \times$ $\{0,1\}^{k}$. A sample $(U, K)$ from the first distribution $\mathcal{D}_{\mathrm{UD}, \mathcal{U}}$ is obtained by sampling $U \stackrel{\$}{\leftarrow}\{0,1\}^{n}$ and $K \stackrel{\$}{\leftarrow}\{0,1\}^{k}$ uniformly at random from the respective domains. A sample $(U, K)$ from the second distribution $\mathcal{D}_{\mathrm{UD}, \mathcal{H}}$ is obtained by sampling $K \stackrel{\$}{\leftarrow}\{0,1\}^{k}$ and then evaluating $\mathrm{H}_{K}$ on a uniformly random bit string, i.e., $\mathcal{U}_{m} \stackrel{\$}{\leftarrow}\{0,1\}^{m}, U \leftarrow \mathrm{H}_{K}\left(\mathcal{U}_{m}\right)$. The success probability of an adversary $\mathcal{A}$ against the undetectability of $\mathcal{H}_{n}$ is defined as:

$$
\operatorname{Succ}_{\mathcal{H}_{n}}^{\mathrm{UD}}(\mathcal{A})=\left|\operatorname{Pr}\left[\mathcal{A}^{\mathcal{D}_{\mathrm{UD}}, \mathcal{U}}=1\right]-\operatorname{Pr}\left[\mathcal{A}^{\mathcal{D}_{\mathrm{UD}}, \mathcal{H}}=1\right]\right|,
$$

where $\mathcal{A}^{\text {dist }}$ denotes that $\mathcal{A}$ has oracle access to some oracle that outputs samples from distribution dist.

The fourth notion we use is pseudorandomness of a function family (PRF). In the definition of the success probability of an adversary against pseudorandomness the adversary gets black-box access to an oracle Box. Box is either initialized with a function from $\mathcal{H}_{n}$ or a function from the set $\mathcal{G}(m, n)$ of all functions with domain $\{0,1\}^{m}$ and range $\{0,1\}^{n}$. The goal of the adversary is to distinguish both cases:

$$
\operatorname{Succ}_{\mathcal{H}_{n}}^{\mathrm{PRF}}(\mathcal{A})=\left|\operatorname{Pr}\left[\operatorname{Box} \stackrel{\$}{\leftarrow} \mathcal{H}_{n}: \mathcal{A}^{\operatorname{Box}(\cdot)}=1\right]-\operatorname{Pr}\left[\operatorname{Box} \stackrel{\$}{\leftarrow} \mathcal{G}(m, n): \mathcal{A}^{\operatorname{Box}(\cdot)}=1\right]\right| .
$$

Subset-Resilient Functions. We now recall the definition of subset resilience from [34]. Let $\mathcal{H}=\left\{\mathrm{H}_{i, t, k}\right\}$ be a family of functions, where $\mathrm{H}_{i, t, k}$ maps a bit string of arbitrary length to an subset of size at most $k$ of the set $[t-1]$. (As for hash functions in the description of SPHINCS we omit the key and assume the used function is randomly selected from a family using the uniform distribution.) Moreover, assume that there is a polynomial-time algorithm that, given $i, 1^{t}, 1^{k}$ and $M$, computes $\mathrm{H}_{i, t, k}(M)$. Then $\mathcal{H}$ is called $\gamma$-subset resilient if the following success probability is negligible for any probabilistic polynomial-time adversary $\mathcal{A}:$

$$
\begin{aligned}
\operatorname{Succ}_{\mathcal{H}}^{\gamma-\mathrm{sr}}(\mathcal{A})=\operatorname{Pr}_{i}\left[\left(M_{1}, M_{2}, \ldots, M_{\gamma+1}\right)\right. & \leftarrow \mathcal{A}\left(i, 1^{t}, 1^{k}\right) \\
\text { s.t. } \mathrm{H}_{i, t, k}\left(M_{\gamma+1}\right) & \left.\subseteq \bigcup_{j=1}^{\gamma} \mathrm{H}_{i, t, k}\left(M_{j}\right)\right]
\end{aligned}
$$

